\title{
Insights Into the Mechanism of Rhizosphere Microorganisms Effecting on Quality Formation of Authentic Angelica Sinensis Under Different Soil Microenvironments
}

\section{Lei Zhu}

Nanjing University of Chinese Medicine

Hui Yan ( $\nabla$ glory-yan@163.com )

Nanjing University of Chinese Medicine

\section{GuiSheng Zhou}

Nanjing University of Chinese Medicine

ChunHao Jiang

Nanjing Agricultural University

Pei Liu

Nanjing University of Chinese Medicine

Guang Yu

Nanjing University of Chinese Medicine

Sheng Guo

Nanjing University of Chinese Medicine

QiNan Wu

Nanjing University of Chinese Medicine

JinAo Duan

Nanjing University of Chinese Medicine

\section{Research Article}

Keywords: Angelica sinensis, rhizosphere microorganism, metabolomics, quality, correlation

Posted Date: January 12th, 2021

DOI: https://doi.org/10.21203/rs.3.rs-134657/v1

License: () (1) This work is licensed under a Creative Commons Attribution 4.0 International License.

Read Full License 
1 Insights into the mechanism of rhizosphere microorganisms effecting

2 on quality formation of authentic Angelica sinensis under different

3 soil microenvironments.

4 Lei Zhu ${ }^{1}$, Hui Yan ${ }^{1, *}$, Gui-sheng Zhou ${ }^{1}$, Chun-hao Jiang ${ }^{2}$, Pei Liu ${ }^{1}$, Guang Yu $^{1}$, Sheng

5 Guo $^{1}$, Qi-Nan $\mathrm{Wu}^{1}$, Jin-ao Duan ${ }^{1, *}$

\section{Abstract}

7 Background: Angelica sinensis (Oliv.) Diels (A. sinensis), the commonly used famous-

8 region Chinese herbs, grew in different geographical locations which were crucial to

9 active components accumulation in medicinal plants. Rhizosphere microbiome played

10 a critical role in plant performance, including nutrient acquisition, growth and

11 development and plant diseases resistance etc., there were still few studies on how

12 microbiome effecting on accumulation of active components of A. sinensis. This study

13 aim to illustrate whether the variations of rhizosphere microbial communities and

*Correspondence: glory-yan@163.com,dja@njucm.edu.cn

${ }^{1}$ National and Local Collaborative Engineering Center of Chinese Medicinal Resources Industrialization and Formulae Innovative Medicine, Jiangsu Collaborative Innovation Center of Chinese Medicinal Resources Industrialization, State Administration of Traditional Chinese Medicine Key Laboratory of Chinese Medicinal Resources Recycling Utilization, Nanjing University of Chinese Medicine, Nanjing, China Full list of author information is available at the end of the article 
14 metabolites profilings of A. sinensis were dependant on soil microenvironments. Soils

15 from the two main A. sinensis producing areas, Gansu and Yunnan Province, were

16 selected for a pot experiment, and then divided into two parts, one part was sterilized

17 and the other did not, planting with Gansu danggui 90-01, allowed to grow for 180 days.

18 After the trial radix A. sinensis were collected for analysis of growth targets and

19 chemical compositions, rhizosphere soils for microbial analyses.

20 Results: The metabolic profiles and rhizosphere microbial communities of A. sinensis

21 grown under different soil microenvironments showed similar variation. The GN

22 (Gansu non-sterilized), YN (Yunnan non-sterilized), GS (Gansu sterilized), and YS

23 (Yunnan sterilized) group were significantly separated. Specifically, compared with

24 Yunnan soil, antagonistic bacteria such as Sphingomonas, Pseudomonas, Lysobacter,

25 Pseudoxanthomonas, etc. were significantly $(p<0.05)$ enriched in Gansu soil. The

26 characteristic constituents of senkyunolide I and ligustilide dimers enriched in GS

27 group has an extremely positive correlation with Pseudomonas parafulva; organic acids

28 (including chlorogenic acid, dicaffeoylquinic acid and 5-feruloylquinic acid) and their

29 ester coniferyl ferulate enriched in YS Group were significantly positively relevant with

30 Gemmatimonadetes bacterium WY71 and Mucilaginibater sp., respectively.

31 Conclusions: Given that soil microenvironments may contribute to growth and active

32 components accumulation of A. sinensis. Further study aimes at exploring the

33 functional characteristics of quality-related bacterias, and finding the main response

34 genes and the interactions between genes and the environments, to elucidate the main 
mechanism of genuine $A$. sinensis quality formation.

36 Key words: Angelica sinensis; rhizosphere microorganism; metabolomics; quality;

37 correlation

\section{Background}

Geo-authentic Chinese medicinal materials refer to traditional Chinese medicine (TCM) that have been selected through long-term TCM clinical practice and are

41 produced in a specific region, with satisfactory curative effect, stable quality and high profile. The radix A. sinensis possessed traditional efficacies including nourishing blood, promoting blood circulation, regulating menstruation and relieving pain, was

44 first recommended in therapy of gynecological diseases [1]. Beyond that, it was 45 generally utilized for protecting the heart, boosting immune system, anti-arrhythmia, 46 and anti-atherosclerosis, etc [2]. A. sinensis was mostly produced in Gansu, Yunnan, 47 Qinghai and other places in China. Min County of Gansu Province has long been 48 acknowledged as its traditional geoherb regions, cool and humid here, abundant rainfall, 49 with mostly fertile cinnamon soil and black soil, the most appropriate material $A$.

50 sinensis to grow. Land plants and soil microorganisms achieved mutual benefit through

51 strong interactions. Plants provided habitats and reliable source of carbon and energy

52 for soil microorganisms which could directly or indirectly facilitate the yield and 53 quality of the host plants and improve the hosts' resistance through increasing 54 effectiveness of soil mineral nutrition, production of phytohormones, degrading 55 phytotoxic compounds, inhibiting soil-borne pathogens, etc [3-4]. Many studies 
showed that plant species, growth and development, host genotypes, geographical location, etc. were the main drivers of rhizosphere microbial diversity [5-7]. Geographical position and cultivation practice were factors determine microbiome variation in field conditions; while under controlled greenhouse conditions, microbial composition varied with soil source and genotype [6]. A very large-scale longitudinal field study comparing changes in microbial communities among 27 maize inbred lines planted in three fields had yielded similar results [7]. The difference was that they found plant genetic effects had a marked impact on the rhizosphere microbiome, surpassing geographic location, plant age and climate events.

Increasing evidences demonstrated that plants-microbe interactions was vital for plant growth and health [8]. At present, the key research for study on genuine TCMs was mainly focused on soil properties and rhizosphere microorganism, important breakthroughs were made in the growth and yield improvement of famous medicinal materials, such as Panax ginseng, Rehmannia glutinosa and P. notoginseng, etc. Changes in rhizosphere microbial communities and accumulation of toxic substance played driving roles in replanting failure of TCMs. For example, soilborne pathogens Fusarium always flourished as plants grew, while antagonistic bacterium Pseudomonas and Bacillus showed opposite trend [9-10]. Although Ilyonectria was generally accepted as the major trigger of ginseng rusty root disease, but recent study found that the oxidation and deposition of rhizosphere Fe, Al and Mn driven by nitrate-dependent Fe (II)-oxidizing bacteria, resulting in rusty root disease [11]. The build-up of Fusarium 
genus and accumulation of toxic diisobutyl phthalate (DiBP), inversely associated with population of Pseudomonas, Bacillus and Burkholderia etc., contributed to negative feedback between the soil and ginseng $[9,12]$. Similarly, phenolic acids accumulated in the rhizosphere of R. glutinosa could restrain the growth of Pseudomonas spp., but had positive effects on mycelial growth, sporulation and toxin production of F. oxysporum [13]. Further research demonstrated that quorum quenching (QQ) bacteria that participated in disruption of beneficial (Pseudomonas spp.) and harmful (Enterobacter spp.) short-chain AHL-mediated QS (quorum sensing) bacteria balance in the rhizosphere, accounting for a key factor of $R$. glutinosa replant diseases [14]. intensity and altitude, suitable for growing in high elevation zones (2000 2500 m). With the decrease of elevation, the radix A. sinensis production and quality were reduced, and more vulnerable to early bolting, resulted in decrease in drug efficacy. In the long-term cultivation practices, the traditional seedling raising manners, wasteland seedling raising, has been retained. Due to the policy of Grain for Green and ecological protection, rotating land seedling and cultivated wasteland seedling have become the main choices, but they are immature to guarantee the quality of seedlings. Little investigation was reported about soil microbial communities of authentic A. sinensis, the unique living environments needs makes it hard on researching ways. Our previous studies had stated that the number of dominant microbial species and the population diversities of bacteria, actinomyces and fungi in the rhizosphere of Gansu province 
were greater than that of other non-geoherb regions, such as Yunnan and Sichuan province. Proteobacteria and bacteroidetes were bacterial dominant phyla throughout the growing season of $A$. sinensis, while fungal dominant phyla varied with growth

101 periods; the plant weight, root length, root diameter, soil $\mathrm{pH}$, rainfall, and climate 102 temperature were the main factors contribute to microbial community composition of

103 A. sinensis [15]. The current study on plant-microbe interactions mainly focused on 104 succession cropping obstacle of medicinal plants, responses of plant-microbe to 105 environmental stress, changes in rhizosphere nutrition conditions, root system biomass 106 and growth; but little support for accumulation of active constituents under different 107 soil microenvironments [16]. There is a pressing need for clarify the mechanism of 108 quality formation of authentic $A$. sinensis, to acquire $A$. sinensis materials in stable 109 quality while enlarging its production. In the present study, a pot experiment was carried out, consisting of four treatments

111 (i) GN, which represent as the soil in pot taken from Gansu province, non-sterilized, (ii)

112 GS, soil in pot taken from Gansu province, sterilized, (iii) YN, soil in pot taken from 113 Yunnan province, non-sterilized, (iiii) YS, soil in pot taken from Yunnan province, 114 sterilized, and four controls (without implants). Using the combination of metabolomics 115 and 16s rRNA pyrosequencing technology, we aimed to address the following questions:

116 (i) Are the rhizosphere microorganisms, and the growth and quality of $A$. sinensis vary

117 in different soil microenvironments? (ii) Whether the growth and quality of $A$. sinensis 118 has a positive response to changes of rhizosphere microorganisms. 


\section{Results}

120

124 137 (amino acids, nucleosides and nucleobases) were compressively analyzed to further 138 explain the inherent differences in quality of $A$. sinensis among groups . The results 139 showed that the contents of medicinal components tested were remarkably $(p<0.05)$

\section{The morphology and quality of $\boldsymbol{A}$. sinensis under different soil conditions}

Regardless of whether the soil was sterilized or not, A. sinensis grown in Gansu soils (including GN and GS group) was significantly $(P<0.05)$ stronger than that of Yunnan soils (including YN and YS group), with more branches and larger diameters (Figure. 1A and B). Compared with non-sterilized soils, plants grown in sterilized soils displayed worse growth as illustrated by the thinner rhizome, reduced number of branches and decreased diameter (Figure. 1C and D). The surviving rate of plants from non-sterilized soils (GN, $0.77 \pm 0.09 ; \mathrm{YN}, 0.63 \pm 0.18 ; n=6)$ was remarkably $(p<0.05)$ higher than that of sterilized soils (GS, $0.48 \pm 0.15$; YS, $0.46 \pm 0.19 ; n=6)$. In addition, we measured the dry weight, diameter, rootlet number and root length of all samples.

The results showed that the average dry weight, root diameter, rootlet number, and root length of plants in GN group were significantly $(p<0.05)$ larger than that of YN group. Compared with GN group, plants in GS group showed noticeably $(p<0.05)$ reduced average dry weight, root diameter and root length. The root diameter of plants in YN group were significantly $(p<0.05)$ higher than in YS group, higher in GS group than YS group (Table. 1).

The major medicinal components (phthalides and organic acids) and nutrients 
140 different among groups. Specifically, the contents of chlorogenic acid and Z-ligustilide

141 of $A$. sinensis grown in Yunnan soil were significantly $(p<0.05)$ higher than that of

142 Gansu soil. Compared to the other three groups, the GN group A. sinensis possessed

143 definitely $(p<0.05)$ higher contents of coniferyl ferulate and butylidenephthalide, and

144 lower content of ferulic acid. The contents of senkyunolide A and butylphthalide in YN

145 group $A$. sinensis were noticeably $(p<0.05)$ greater than other groups. The contents of

146 senkyunolide I and senkyunolide H in YS group $A$. sinensis were significantly $(p<0.05)$

147 lower than other groups. However, both senkyunolide I and senkyunolide H showed no

148 significant difference in the A. sinensis samples from GN, YN and GS groups (Table.

$1492)$.

150 Our previous publications indicated that $A$. sinensis samples have high contents of

151 nutritive component including nucleosides, nucleobases and free amino acids [17].

152 Additionally, our previous reports also showed that the contents of nucleosides and

153 nucleobases were no significant difference between different groups, while the contents

154 of amino acids had certain differences. The mean content of arginine accounted for

$15586.02 \%$ of the total amino acids, much higher than other relatively abundant ones, such

156 as $\gamma$-aminobutyric acid (4.78\%), glutamine (2.18\%) and proline (1.07\%). Compared

157 with the GN or GS group, the concentrations of arginine, $\gamma$-aminobutyric acid,

158 glutamine and proline in $\mathrm{YN}$ or YS group displayed a decreasing tendency. The changes

159 of chemical components among groups might be explained by differences in soil

160 microenvironments. 


\section{microenvironments}

163

The quality of medicinal plants was not focused on a single compound, but

164 involved in a special mixture of diverse, biochemistry related and unrelated compound

165 groups [18]. UPLC with high-resolution mass spectrometry provided powerful

166 functions for isolating and profiling a large amount of many kinds of metabolites in

167 different matries. In this study, a simple, rapid and sensitive UPLC-UV-QTOF-MS/MS

168 method was developed to evaluate the variation of the metabolites in A. sinensis

169 samples under different soil conditions. Principal components analysis (PCA) showed

170 that metabolic profiles among groups were significantly $(P<0.05)$ separated, all

171 samples clustered into four groups, GN, YN, GS and YS groups. Moreover, the GS and

172 YS samples were found to be gathered close to GN samples, especially GS samples,

173 but far away from $\mathrm{YN}$ samples (Figure. 2A). The $\mathrm{R}^{2} \mathrm{X}, \mathrm{R}^{2} \mathrm{Y}$ and $\mathrm{Q}^{2} \mathrm{Y}$ of the OPLS-DA

174 model were $0.667,0.966$ and 0.851 , respectively. The permutation results verified the

175 stability and reliability of this OPLS-DA model. The hierarchical clustering heatmap

176 showed similar gathering patterns of molecular features detected within groups and

177 differences in molecular features detected between groups, which was consistent with

178 the PCA clustering pattern (Figure. 3A).

179 In the UPLC-QTOF-MS/MS analysis, both positive and negative ion modes were

180 employed for qualitative identification. A total of 44 metabolites were identified by

181 chemical standards or tentatively identified by comparing their quasi-molecular ions, 
182

183

184

185

186

187

188

189

190

191

192

193

194

195

196

197

198

199

200

201

202

empirical molecular formulas, and/or fragment ions with those of known chemicals (Supplementary Table S1) [19]. It was workable to select markers that contributed greatly to the grouping by setting the Variable Importance for Projection (VIP) value greater than 1.5 and the $p$-value less than 0.05 in the Moderated t-Test. In general, compared to YN and YS groups, the contents of ligustilide dimers increased in plants from GN and GS groups, while the content of chlorogenic acid was opposite. More concretely, compared with YN group, the plants of GN group was higher in ligustilide dimers, while the contents of unknown [19], senkyunolide A and chlorogenic acid declined. Secondly, compared with YS group, the plants of GS group displayed higher contents of ligustilide dimers, Z-6,7-epoxyligustilide and senkyunolide I, while the contents of both chlorogenic acid and dicaffeoylquinic acid showed decreasing trend.

It was noticed that the plants in non-sterilized groups had higher levels of ligustilide dimers than that of the sterilized groups (Supplementary Fig. S1, Supplementary Table S2).

Annotation and community composition of rhizosphere bacteria under different soil microenvironments

The V4 region of 16S rRNA gene of total 36 samples were amplified using PCR and sequenced using the IonS5 ${ }^{\mathrm{TM}} \mathrm{XL}$ platform. An average of 80,118 clean reads per sample was obtained, the high-quality reads were clustered using $>97 \%$ sequence identity into 10,583 OTUs, and then annotated using Silva132 database. A total of 65 kinds of the OTUs were annotated at the phylum level, accounting for $87.60 \%$ of all 
203 OTUs; and 721 kinds of the OTUs were annotated at the genus level . In the phylum 204 level, we found that Proteobacteria (relative abundance of 55.38\%), Bacteroidetes $205(10.65 \%)$ and Acidobacteria (7.26\%) were the dominant species; in the genus level, the 206 roots were dominated by Stenotrophomonas (9.97\%), Rhodanobacter $(4.19 \%)$ and 207 Lactobacillus (1.48\%).

$208 \alpha$-diversity refers to the microbial diversity of each sample, similar conclusions 209 were drawn by calculating the Chao1, Shannon's diversity index and Simpson diversity 210 index, etc (Figure. 4A and B). The results showed that the community diversity of the 211 non-sterilized groups were higher than that of the sterilized groups, and the community 212 diversity in the Gansu groups were higher than that in Yunnan groups, but there was 213 little difference between the planting groups and the corresponding non-planting groups.

214 ANOSIM showed that the difference between groups were significantly $(p<0.05)$

215 greater than that of within groups, indicating that the results were reliable. Therefore, 216 these results indicated that the diversity of the bacterial communities of $A$. sinensis was 217 determined by the soil microenvironments, and the plant effect had little effect.

218 PCoA based on weighted UniFrac (WUF) distance metric showed that the 219 community compositions of the planting groups and corresponding non-planting 220 groups were highly similar. The sterilized and non-sterilized samples separated along 221 the first axis, PC1 explained $38.65 \%$ of the total variation, indicating that indigenous 222 bacteria were the most important factor affecting the rhizosphere bacterial community.

223 The Gansu and Yunnan samples could be clearly distinguished on the second axis, PC2 
explained $23.35 \%$ of the total variation, indicating that geographical location were the

225 second largest factor accounting for the changes of rhizosphere bacterial community.

226 However, when using an unweighted UniFrac (UUF) distance, geographic location had

227 the largest source of variation in the tested factors, and indigenous bacteria described

228 the second largest source of variation (Figure. 2B).

arithmetic mean (UPGMA) clustering (Figure. 2C). The hierarchical clustering based

231 on a UUF distance metric showed complete clustering of samples in GN, GS, YN, and

232 YS groups. But the planting groups and the corresponding non-planting groups

233 gathered together, indicating that plant effect made little difference to the overall

234 community composition. The GS and the YS samples first gathered into a branch, then

235 the YN samples, and finally the GN samples. It showed that GN group differed greatly

236 with the other three groups. In order to support the above analysis statistically, we used

237 ADONIS and MRPP, etc. to perform statistical tests on samples from different groups,

238 similar conclusion was derived.

239 Comparison of rhizosphere bacteria composition between different groups

240 In order to fully understand the OTU distribution among groups, we constructed

241 Venn diagrams, and then calculated the proportion of uniquely identified OTUs in each

242 group and OTUs shared between different groups. Among them, the proportion of

243 OTUs shared between the GN and GNck group, YN and YNck group, GN and YN

244 group, GS and YS group, GN and GS group, YN and YS group accounted for 48.74\%, 
246 indicated the high similarity of the microbial community structure between the planting

247 groups and corresponding non-planting groups. We then drew a Venn diagram for all

248 planting groups, including GN, GS, YN, and YS group, it was shown that the number

249 of unique OTUs belong to the GN group were the largest, with 1692 species; and the

250 YS group was the least, with 226 species. The same phenomena were recorded in all

251 non-planting groups.

252 At the phylum level, we selected the top 12 most abundant phylum for each sample

253 or group to generate an accumulative column diagram of the relative abundance of all

254 species. It could be seen that the microbial community was dominated by

255 Proteobacteria, Bacteroidetes, and Acidobacteria. Compared to the non-sterilized

256 groups, a notable increase was observed in the relative abundance of Proteobacteria

257 and Bacteroidetes of all sterilized groups, while the relative abundance of

258 Acidobacteria, Actinobacteria, and Chloroflexi decreased significantly $(p<0.05)$.

259 Compared with the GN group, the relative abundance of Chloroflexi and

260 Planctomycetes was significantly $(p<0.05)$ higher in YN group, while the relative

261 abundance of Acidobacteria displayed significant $(p<0.05)$ reduction; Compared with

262 the YS group, the GS group significantly $(p<0.05)$ increased the relative abundance of

263 Gemmatimonadetes, Actinobacteria, and Chloroflexi, while the relative abundance of

264 Proteobacteria was opposite. The discrepancy of microbial community between

265 planting groups and corresponding non-planting groups was insignificant $(p>0.05)$. 
266 Figure. 4C shows the distribution of phylum level.

267 At the genus level, a total of 721 taxa were identified from all 36 samples. The 268 roots of A. sinensis were preferentially colonized by Stenotrophomonas, 269 Rhodanobacter and Lactobacillus. The heatmap visualization with hierarchical 270 clustering of top 35 most abundant genera showed (Figure. 4D) that there were distinct 271 differences in microbial community structure and relative abundance of most 272 predominant genera between different groups. Compared with the Yunnan group, the 273 relative abundance of Lysobacter and Brevundimonas in Gansu group increased 274 significantly $(p<0.05)$, while the relative abundance of Rhodanobacter and 275 Gemmatimonas decreased significantly $(p<0.05)$. In addition, the relative abundance 276 of Sphingomonas in the GN group was higher than that of YN group, while the opposite 277 was true for relative abundance of Mucilaginibacter. Secondly, the relative abundance 278 of Pseudomonas in GS group was higher than that of YS group; while the relative 279 abundance of Ramlibacter and Sphingomonas were opposite. It was remarkable that the 280 relative abundance of Sphingomonas in all non-sterilized groups was significantly $(p<$ 2810.05 ) higher than the corresponding sterilized groups.

282 Putative mechanistic associations between microbes and quality-linked 283 metabolites.

284 The secondary metabolites of A sinensis were mainly composed of phthalides, 285 organic acids, and polysaccharides, among which phthalides were the main bioactive 286 ingredients, with a wealth of structural diversity [2,20]. In the previous study, we found 

Brevundimonas were significantly $(p<0.05)$ enriched in GS group. The contents of ligustilide dimer and senkyunolide I in GS group were significantly $(p<0.05)$ higher than that in YS group, and were positively correlated with Pseudomonas parafulva, positively correlated with $P$. parafulva (Spearman $\mathrm{r}=0.82)($ Supplementary Table S3). It was speculated that the high abundance of $P$. parafulva in the GS group contribute to

295 the accumulation of phthalides and was beneficial to the growth and quality formation 296 of $A$. sinensis.

Some ferulic acid derivatives such as ferulic acid ester have stronger biological 298 activity and lower toxicity. Organic acids and their esters were significantly $(p<0.05)$ 299 enriched in YS group. It showed that chlorogenic acid, dicaffeoylquinic acid and 5300 feruloylquinic acid were positively correlated with Gemmatimonadetes bacterium 301 WY71 (average Spearman $r>0.8$ ); coniferyl ferulate was positively correlated with 302 Mucilaginibacter sp. (Spearman r=0.85) (Figure. 3B) (Supplementary Table S3) [21]. 303 Mucilaginibacter was widely present in the rhizosphere soil of Angelica medicinal 304 plants. For example, M. herbaticus sp nov. [22], M. polysacchareus sp nov. [23], and 305 M. angelicae sp nov. [24] were isolated respectively from the rhizosphere, root surface 306 of A. sinensis, and rhizosphere of A. polymorpha Maxim. It was speculated that the high 307 abundance of Mucilaginibacter in YS soil might be related to the accumulation of 
ferulic acid derivatives.

\section{Discussion}

\section{Driving factors of $\boldsymbol{A}$. sinensis's quality formation under different soil conditions}

311 Considerable researches have indicated that the quality of Chinese drugs showed

312 a significantly geographical differences, this might due to variation of altitude, climate,

313 soil properties and rhizospheric microorganisms, etc. Our study showed that soil

314 microenvironments was a significant factor contribute to the quality formation of $A$.

315 sinensis. The tradition holds that the higher the contents of ferulic acid and $Z$-ligustilide,

316 the better the quality of the radix A. Sinensis. Compared with the YN and GS group,

317 GN group displayed significantly $(p<0.05)$ larger dry weight, root diameter, and root

318 length (Table. 1); and the contents of chlorogenic acid and Z-ligustilide in Yunnan groups

319 were significantly $(p<0.05)$ higher than that of Gansu groups (Table. 2). Compared

320 with Yunnan groups, the content of senkyunolide I, butylidenephthalide, ligustilide

321 dimers increased in Gansu groups, while ferulic acid displayed decreasing trend (Table.

322 1, Supplementary Table S2). Zhang et al found that the contents of ferulic acid and

323 levistolide A in non-geoherbs regions were higher than that of geoherbs regions, while

324 the opposite was true for senkyunolide I and butylidenephthalide [19], which supported

325 our conclusions. Similar results had uncovered through a large-scale field investigation,

326 the difference was that the root length and head diameter showed negative correlation

327 to ferulic acid, as well as root length to Z-ligustilide [25]. Therefore, it was inaccurate

328 to estimate the quality of radix A.sinensis from their appearance traits. 
330 coumalic acid, ferulic acid, vanillic acid, 4-hydroxybenzoic acid, and syringic acid are

331 found in root exudates of $P$. ginseng, $R$. glutinosa, and Pinellia ternata [26]. Imperatorin,

332 vanillin, and ferulic acid were reported as possible allelopathic autotoxic substances in

333 rhizosphere soil of A.sinensis, which could obviously inhibit the growth of its seedlings

334 [27]. The increased content of ferulic acid in plants of Yunnan groups, indicating the

335 larger accumulation of allelochemicals in the Yunnan soils, which led to the deteriorated

336 growth of $A$. sinensis. Similarly, higher content of ferulic acid in sterilized groups than

337 in non-sterilized groups may also be the reason for the weak growth of plants in the 338 sterilized groups.

339 Effect of soil type on the composition of rhizosphere bacteria of $\boldsymbol{A}$. sinensis

340 A continental-scale studies of soil bacterial populations showed that the richness

341 and diversity of soil bacterial communities differed by ecosystem type, among which

342 soil $\mathrm{pH}$ explained the largest source of variation [28]. For example, compared to loessial

343 soil and continuous cropping soil, polysaccharides and glucose increased significantly

344 in Dioscorea opposita Thunb. growing in sandy soil, moreover, the decreased soil $\mathrm{pH}$

345 might contribute to the high level of galacturonic acid from continuous cropping soil

346 [29]. The soil of Gansu was slightly alkaline, while the soil of Yunnan was weakly

347 acidic, different soil $\mathrm{pH}$ caused significant differences in the rhizosphere and root-

348 associated microbiomes of plant. It was shown that the relative abundance of

349 Sphingomonas, Lysobacter and Pseudoxanthomonas in the GN group was higher than 
350 that of YN group; while the relative abundance of Rhodanobacter, Gemmatimonas and

351 Mucilaginibacter decreased significantly $(p<0.05)$ (Figure. 4D). Compared with the 352 YS group, the relative abundance of Pseudomonas, Lysobacter and 353 Pseudoxanthomonas increased in GS group; while Rhodanobacter, Ramlibacter, 354 Dyella were opposite (Figure. 4D). Wu et al found that the relative abundance of 355 Lysobacter, Pseudoxanthomonas, Pseudomonas and Burkholderia were significantly $356(p<0.05)$ increased in the newly cultivated soil of $R$. glutinosa compared to continuous 357 monocropping soil [30]. Similarly, the abundance of beneficial bacteria such as 358 Arthrobacter, Burkholderia, Rhodanobacter, and Sphingobacterium displayed a 359 negatively correlation to the accumulation of toxic diisobutyl phthalate (DiBP) in 360 monocropping soil of $P$. ginseng [12]. We speculated that genus Lysobacter, 361 Pseudomonas, and Burkholderia etc. might be core microbes for radix or rhizoma 362 medicinal herbs.

363 Previous studies demonstrated that Sphingomonas [31], Lysobacter [32-33] and 364 Pseudoxanthomonas [34-35] had functions of enhancing resistance to diseases and 365 insect pests, and degrading harmful substances in the soil. Sphingomonas could also 366 promote plant absorption by secreting carbohydrate into the rhizosphere [31]. 367 Lysobacter strains had strong antagonistic activity against a series of pathogens 368 (including R. solani, F. oxysporum and X. carnpestris, etc.) [32]. Genus Pseudomonas 369 was proved to be a group of plant growth promoting rhizobacteria (PGPR) [36-37]. For 370 instance, Pseudomonas was found to be a key PGPR in the rhizosphere of garlic 
371 regardless of growth periods, soil types and agricultural practices, the constructed six

372 Pseudomonas strains displayed strong plant growth promoting effects [38]. These

373 results indicated that the stronger growth characteristics of $A$. sinensis in Gansu group

374 may be related to the increased abundance of these antagonistic microorganisms in the

375 rhizosphere.

376 Effects of indigenous microorganisms on rhizosphere bacterial communities and

377 growth of $A$. sinensis

378 Many years of planting sole crop, such as grain crops, vegetable crops, medicinal

379 plants, etc., often bring about widespread continuous cropping problems. Speculation

380 had it that soil sterilization could change the physical structure and microbiological

381 properties of soil, which resulted in increased nutrient release, and inhibition of soil

382 pathogens, thus promote plant growth. For example, A. sinensis from sterilized replant

383 soil exhibited higher plant performance and enhanced activities of active oxygen

384 scavenging enzymes, which might cause by the increased number and diversity of

385 culturable microbial populations and bacterial functional groups [39]. While soil

386 sterilization could relieve the soil succession cropping failure, it not only removed

387 indigenous microbes, including some pathogens, but could also destroy the possible

388 beneficial effects of soil microbes to host plants [40]. Some researches indicated that

389 indigenous microorganisms were vital on plant growth and agricultural productivity,

390 and it's possible effects on plant secondary metabolism [41]. For example, inoculation

391 with PGPR B. flexus could alleviate host salinity stress damage by modifying its 
392 physiological and biochemical state, such as enhancement of photosynthesis, osmotic 393 regulator contents, antioxidant enzymes activities, and regulation of $\mathrm{Na}^{+} / \mathrm{K}^{+}$ 394 homeostasis [42].

395 Our study showed that plants grown in sterilized soil displayed worse growth and 396 decreased surviving rate, it might because the soil we used were newly cropped (Table. 397 1), which confirmed the prediction that indigenous microorganisms were essential to 398 satisfy the plant growth and development. Likewise, Glycyrrhiza uralensis grown in 399 sterilized soil displayed poorer plant growth and photosynthesis, inoculation with AM 400 fungi in sterilized soil compensated for the absence of indigenous microbial 401 communities, leading to increased plant growth and glycyrrhizin and liquiritin contents 402 [40]. We also found that compared with the GS group, the relative abundance of 403 Sphingomonas increased significantly $(p<0.05)$ in the GN group; while Ramlibacter, 404 Gemmatimonas and Brevundimonas were opposite (Figure. 4D). The relative 405 abundance of Sphingomonas and Bradyrhizobium in YN group were higher than that 406 of YS group; while Rhodanobacter, Ramlibacter and Dyella decreased significantly ( $p$ 407 <.05) (Figure. 4D). Bradyrhizobium was also a known PGPR that can produce plant 408 hormones and QS metabolites to regulate the symbiotic relationship with the host and 409 adapt to environmental changes [43]. QS-deficient (unable to produce cinnamoyl-HSL) 410 mutant strains exhibited significant decreased colonization ability and reduced plant 411 growth compared to wildtype strain [44]. The high abundance of beneficial bacteria, 412 such as Sphingomonas and Bradyrhizobium, in the rhizosphere of non-sterilized groups 
413 accounted for better growth and quality of A. sinensis in the unsterilized groups.

414 Potentially mechanistic associations between rhizosphere microbes and 415 metabolites

416 Many plant endophytes are able to synthesize a variety of secondary metabolites,

417 same or similar to their host, as well as some new compounds, some of them show good

418 biological activities, which help to quality improvement of medicinal plants [45]. For

419 example, inoculation with endophytes isolated from the alkaloid-rich genotype of

420 Catharanthus roseus, can remarkably improve ajmalicine and serpentine contents in

421 roots of low alkaloid yielding genotype [46]. The small moleculars secreted by roots

422 such as amino acids, organic acids, sugars and secondary metabolites can specifically

423 promote the growth of some soil microorganisms, causing the migration of soil

424 microbial communities [47]. For instance, Falciphora oryzae colonized Arabidopsis

425 roots by sensing signalling molecules from its roots, and then promoted the lateral root

426 growth of Arabidopsis through biosynthesis of indole derivatives [48]. We speculated

427 that rhizosphere microbes can also directly or indirectly envolved in and influence the

428 growth and metabolism process of hosts. For example, PGPR, mycorrhizal fungi and

429 Actinomycetes from Platycodon grandiflorum [49], P. ginseng [50], and Curcuma

430 longa [51] can significantly promote plant growth and resistance. We observed that the

431 metabolite profiling of $A$. sinensis and microbial community composition in the

432 rhizosphere soil had similar variation patterns among groups. Therefore, it was

433 concluded that there existed a certain relationship between the secondary metabolites 
of $A$. sinensis and its rhizosphere microorganisms.

Our research demonstrated that the higher contents of ligustilide dimers and senkyunolide I in GS group was positively correlated with P. parafulva, P. maxicana, and B. bullata (average Spearman $r>0.5$ ), and the higher contents of chlorogenic acid, dicaffeoylquinic acid, 5-feruloylquinic acid in YS group were positively correlated with Gemmatimonadetes bacterium WY71 (average Spearman r $>0.8$ ); similarly, coniferyl ferulate to Mucilaginibacter sp. (Spearman $\mathrm{r}=0.85$ ) (Supplementary Table S3). A positive association between a metabolite and species may indicate that the metabolite promotes the growth of the that species, or that the species produces that metabolite [52]. Studies have shown that certain microorganisms can promote the accumulation of metabolites through up-regulating the expression of genes involved in the synthesis of secondary metabolites, activating the host's defense responses, and synthesis of key enzymes that convert precursors into effective constituents [53]. For example, Pseudomonas sp. and Pantoea sp. in the rhizosphere of Salvia miltiorrhiza could stimulate the plant growth and synthesize of major phenolic acids, which due to its production of the abundant types and contents of phytohormones [54]. Inoculation of Trichoderma asperellum significantly increased the artemisinin concentration and dry weight of Artemisia annua L. leaves by up-regulating the expression of artemisinin biosynthesis key enzyme genes $H M G R 1, F P S, A D S, C Y P 71 A V 1, C P R, D B R, D X S 1$ and DXR1 [55]. Dark Septate Endophytes (DSE) could establish symbiosis with liquorice, promoted its plant $\mathrm{N}$ and $\mathrm{P}$ absorption, biomass, as well as accumulations of 
456 of antibacterial secondary metabolites such as lipopeptides, pyridine, benazine and 457 hydrogen cyanide, and exhibited an excellent antagonism to plant pathogens of rice and 458 soybeans (including Rhizoctonia solani, Xanthomonas axonopodis and Burkholderia 459 glumae.) [57-58]. A next step is to further identify whether the observed population 460 structure was stable over time or correlated with changes in metabolomic or 461 microbiome composition in A. sinensis, and to distinguish the plant genes that associate 462 with these taxa and to better characterize their functions [7].

\section{Conclusion}

In summary, this study analysed changes of metabolite profilings and rhizosphere microbial communities of $A$. sinensis under different soil microenvironments, and

466 provides some opinions into mechanism of rhizosphere microorganisms effecting on

467 quality formation of authentic A. sinensis. The results proved that the surviving rate and 468 growth status of $A$. sinensis in Gansu soils was stronger than that of Yunnan soils, higher 469 in unsterilized groups than sterilized groups. High abundance of beneficial bacteria 470 Sphingomonas, Pseudomonas, Lysobacter, Pseudoxanthomonas in Gansu soils and the 471 enhanced growth characteristics of A. sinensis from Gansu soils revealed that the 472 rhizosphere microbiome was necessarily required by plants for normal growth and 473 development. Further more, ligustilide dimers enriched in GS group presented a notable 474 positive correlation with the relative abundance of $P$. parafulva; organic acids enriched 475 in YS group were positively correlated with the relative abundance of 
477 foundation for further research of the relationship between rhizosphere microbes and 478 growth and quality formation of $A$. sinensis as well as its mechanism in order to 479 essentially elucidate the mechanism of the connection of microorganism and growth of 480 medicinal plants.

481 Materials and Methods:

\section{Pot experiment site and design}

The tested soils were taken from the experimental base of the Min Conty Medcinal

Plants Growing Technology Extension Centre (34²2'30" N, 10453'20" E; black soil, $\mathrm{pH} \approx 8.0$ ) and the Medcinal Plants Research Institute Yunnan Academy of Agricultural

Sciences $\left(26^{\circ} 28\right.$ '42" N, 1004'34" E; red soil, pH $\left.\approx 6.5\right)$, where Vicia faba L. was the previous crop. The soil samples then transported into Shili Town of Min County, Gansu

488 Province, where the average annual rainfall was $635.0 \pm 17.4 \mathrm{~mm}$, the annual average temperature was $5.43 \pm 0.71{ }^{\circ} \mathrm{C}$, and the yearly mean sunshine duration was $2154.3 \pm$ $68.7 \mathrm{~h}$. After sieving $(2 \mathrm{~mm})$, the soil samples were then divided into two parts, one part was sterilized by autoclaving at $121{ }^{\circ} \mathrm{C}$ for 4 hours; the other part did not. The $A$. sinensis seedlings (Gansu danggui 90-01) were provided by Min County Xizhai town

493 Zhucai herbs Planting Professional Cooperative, and were transplanted during the local 494 traditional planting period. The experiment set up eight groups, including four 495 experimental groups (GN, GS, YN, YS) and four control groups (GN-ck, GS-ck, YN496 ck, YS-ck). The soil without implants served as a control group, six repeats of each 
group. mixed soil up to $4 \sim 6 \mathrm{~cm}$ lower the container mouth, layered compacted. 8 seedlings of pests and diseases in time. When drought occurred $2 \mathrm{~cm}$ below the topsoil, irrigating each pot with the same amount of water. During 2018, A. sinensis plants were allowed to grow for 180 days. Carefully uprooted the fresh plants from the soil and slightly

507 shaken to remove loosely attached soil [30]. The rhizosphere soil tightly attached to

508 tuberous roots was collected. The fresh plants and soil samples were then transferred to 509 the laboratory in an ice cooler and stored at $-80{ }^{\circ} \mathrm{C}$ until analysis.

\section{DNA extraction, PCR amplification and sequencing}

A total of 36 soil samples collected from the 8 groups of the pot experiment (GN,

512 GS, YN, YS, GN-ck, GS-ck, YN-ck, YS-ck) were sent to 100015 (China) Beijing

513 Novogene Biotech Co., Ltd. to perform 16S rRNA gene amplicon sequencing through

514 Ion S5 ${ }^{\mathrm{TM}}$ XL platform. Use CTAB or SDS method to extract genomic DNA, use agarose

515 gel electrophoresis to detect the purity and concentration of DNA, and then dilute it

516 with sterile water to $1 \mathrm{ng} \cdot \mu \mathrm{L}^{-1}$. The variable region $\mathrm{V} 4$ of the bacterial $16 \mathrm{~S}$ rRNA gene 517 was amplified with degenerate PCR primers 515F and 806R (Walters et al. 2016). The 
samples were separated on a $2 \%(\mathrm{w} / \mathrm{v})$ agarose gel, and mixed together in

519 equivalent amounts according to the concentration of PCR products. The mixed PCR

520 products were purified using the GeneJET ${ }^{\mathrm{TM}}$ Gel Extraction Kit (Thermo Scientific).

521 Briefly, 16S rRNA gene amplicons were extracted by Ion Plus Fragment Library Kit 48

522 rxns (Thermo Scientific) according to the manufacturer's instructions. The built DNA

523 amplicon library was sequenced on the Ion S5 ${ }^{\mathrm{TM}}$ XL platform after Qubit quantification

524 and examination of genomic libraries.

525 Data processing and analysis

526 The raw sequencing data from Ion AmpliSeq were processed by using Cutadapt

527 (V 1.9.1) (Martin M., 2011). Remove the barcode and primer sequence for preliminary

528 quality control to obtain raw reads. The chimera sequences were detected and deleted

529 from the dataset by using UCHIME algorithm to finally get clean reads. The clean reads

530 were clustered into operational taxonomic units (OTUs) at a sequence similarity level

531 of $97 \%$ by using Uparse software (v7.0.1001). Taxonomic annotation through the

532 SSUrRNA database was performed by Mothur (version 1.25.1)and SILVA132

533 (http://www.arb-silva.de/). Use MUSCLE (V 3.8.31) software to perform multiple

534 sequence alignment to obtain the phylogenetic relationship of all OTUs sequences. Use

535 QIIME (V 1.9.1) and R software (V 2.15.3) for sample diversity analysis. $\alpha$-diversity

536 was calculated considering OTU tables. Spearman rank correlation analysis was

537 performed by $\mathrm{R}$ using the vegan packages.

538 Multi-index comprehensive evaluation of the chemical composition of $A$. sinensis 
$54125^{\circ} \mathrm{C}$ ) with $4 \mathrm{~mL} 70 \%$ methanol for 45 mins in a $50 \mathrm{~mL}$ glass-stoppered conical flask.

542 The samples were centrifuged (12000 r/min, $10 \mathrm{mins})$, and the supernatants were

543 injected directly onto a A Thermo Syncronis $\mathrm{C}_{18}(2.1 \mathrm{~mm} \times 100 \mathrm{~mm}, 1.7 \mu \mathrm{m})$ column.

544 The mobile phase consisted of A ( $0.1 \%$ formic acid $)$ and B (acetonitrile). The optimized 545 elution conditions were as follows: $0 \sim 2 \mathrm{~min}, 5 \% \sim 10 \% \mathrm{~B} ; 2 \sim 10 \mathrm{~min}, 10 \% \sim 40 \% \mathrm{~B}$; $54610 \sim 14 \mathrm{~min}, 40 \% \sim 58 \% \mathrm{~B}$; 14 20 $\mathrm{min}, 58 \% \sim 90 \% \mathrm{~B} ; 20 \sim 22 \mathrm{~min}, 90 \% \sim 90 \% \mathrm{~B} ; 22 \sim 22.5$ $547 \min , 90 \% \sim 5 \% \mathrm{~B} ; 22.5 \sim 24 \mathrm{~min}, 5 \% \sim 5 \% \mathrm{~B}$. The flow rate is $0.4 \mathrm{~mL} \cdot \mathrm{min}^{-1}$, 548 column temperature remained at $35^{\circ} \mathrm{C}$, and the injection volume was $2 \mu \mathrm{L}$. 549 Mass spectrometric detection was carried out on a Waters ACQUITY ${ }^{\text {TM }}$ Synapt Q-TOF 550 mass spectrometer equipped with an electrospray ionization (ESI) source. ESI-MS 551 spectra were obtained in both positive $\left(\mathrm{ESI}^{+}\right)$and negative $\left(\mathrm{ESI}^{-}\right)$ion modes by scanning 552 from 100 to $1000 \mathrm{Da}$. The optimized conditions referenced to published articles in 553 Chemistry Central Journal [59].

554 Method 2: preparation of Nucleosides, Nucleobases and Amino Acids analysis 555 samples and standard solutions for qualitative and quantitative analysis.

556 Referenced to published articles in Molecules [17]. A mixed standard stock 557 solution of 26 reference compounds was prepared in methanol/water $(9: 1, \mathrm{v} / \mathrm{v})$, the 558 concentration of which was thymine $4.1 \mu \mathrm{g} \cdot \mathrm{mL}^{-1}$, thymidine $3.8 \mu \mathrm{g} \cdot \mathrm{mL}^{-1}, 2^{\prime}-$ 559 deoxyguanosine $3.7 \mu \mathrm{g} \cdot \mathrm{mL}^{-1}$, cytidine $4.4 \mu \mathrm{g} \cdot \mathrm{mL}^{-1}, 2^{\prime}$ - deoxyuridine $5.3 \mu \mathrm{g} \cdot \mathrm{mL}^{-1}$, 
cytidine-5'-monophosphate $4.1 \mu \mathrm{g} \cdot \mathrm{mL}^{-1}$, guanine $3.8 \mu \mathrm{g} \cdot \mathrm{mL}^{-1}$, ornithine $3.6 \mu \mathrm{g} \cdot \mathrm{mL}^{-1}$,

561 leucine $15.0 \mu \mathrm{g} \cdot \mathrm{mL}^{-1}$, iso-leucine $15.0 \mu \mathrm{g} \cdot \mathrm{mL}^{-1}$, phenylalamine $15.3 \mu \mathrm{g} \cdot \mathrm{mL}^{-1}$,

562 tryptophan $15.8 \mu \mathrm{g} \cdot \mathrm{mL}^{-1}$, aminobutyric acid $19.0 \mu \mathrm{g} \cdot \mathrm{mL}^{-1}$, methionine $17.0 \mu \mathrm{g} \cdot \mathrm{mL}^{-1}$,

563 proline $18.5 \mu \mathrm{g} \cdot \mathrm{mL}^{-1}$, valine $17.2 \mu \mathrm{g} \cdot \mathrm{mL}^{-1}$, trans-4-hydroxy-L-proline $21.0 \mu \mathrm{g} \cdot \mathrm{mL}^{-1}$,

564 glutamincacid $15.7 \mu \mathrm{g} \cdot \mathrm{mL}^{-1}$, glutamine $13.8 \mu \mathrm{g} \cdot \mathrm{mL}^{-1}$ and asparagine $23.8 \mu \mathrm{g} \cdot \mathrm{mL}^{-1}$,

565 tyrosine $15.3 \mu \mathrm{g} \cdot \mathrm{mL}^{-1}$, threonine $17.3 \mu \mathrm{g} \cdot \mathrm{mL}^{-1}$, lysine $18.5 \mu \mathrm{g} \cdot \mathrm{mL}^{-1}$, serine $15.5 \mu \mathrm{g} \cdot \mathrm{mL}^{-}$

$566{ }^{1}$, citrulline $17.3 \mu \mathrm{g} \cdot \mathrm{mL}^{-1}$, arginine $32.3 \mu \mathrm{g} \cdot \mathrm{mL}^{-1}$. The working standard solution for the

567 calibration curves were prepared by diluting the mixed standard solution with $10 \%$

568 methanol at different concentrations.

569 Method 3: Preparation of organic acids and phthalides analysis samples and

$570 \quad$ mixed control solutions.

$5710.2 \mathrm{~g}$ dried powder (40 mesh) was accurately weighed and extracted in the

572 ultrasound assisted condition at $25^{\circ} \mathrm{C}$ with $20 \mathrm{~mL}$ methanol for 40 mins in a $50 \mathrm{~mL}$

573 glass-stoppered conical flask. After centrifugation (13,000 r/min, $10 \mathrm{mins})$ and filtration

$574(0.22 \mu \mathrm{m}$ membrane filter), the supernatants were injected into the UPLC system for

575 analysis. A mixed standard stock solution of 9 reference compounds was prepared in

576 methanol/water $(9: 1, \mathrm{v} / \mathrm{v})$, the concentration of which was chlorogenic acid 24.1313

$577 \mathrm{mg} \cdot \mathrm{L}^{-1}$, ferulic acid $15.8838 \mathrm{mg} \cdot \mathrm{L}^{-1}$, senkyunolide I $7.5600 \mathrm{mg} \cdot \mathrm{L}^{-1}$, senkyunolide $\mathrm{H}$

$5783.0208 \mathrm{mg} \cdot \mathrm{L}^{-1}$, coniferyl ferulate $18.0340 \mathrm{mg} \cdot \mathrm{L}^{-1}$, senkyunolide A $5.3025 \mathrm{mg} \cdot \mathrm{L}^{-1}, \mathrm{n}$ -

579 butylphthalide $199.9788 \mathrm{mg} \cdot \mathrm{L}^{-1}$, Z-ligustilide $17.9196 \mathrm{mg} \cdot \mathrm{L}^{-1}$, butylidenephthalide

$58015.0 \mathrm{mg} \cdot \mathrm{L}^{-1}$. The working standard solution for calibration curves were prepared by 
581 diluting the mixed standard solution with methanol at different concentrations. The 582 separation was performed on a Thermo Syncronis $\mathrm{C}_{18}(2.1 \mathrm{~mm} \times 100 \mathrm{~mm}, 1.7 \mu \mathrm{m})$ 583 column; mobile phase consisted of A ( $0.1 \%$ formic acid) and B (acetonitrile) with a 584 gradient elution: $0 \sim 2 \mathrm{~min}, 5 \% \sim 10 \% \mathrm{~B} ; 2 \sim 10 \mathrm{~min}, 10 \% \sim 40 \% \mathrm{~B} ; 10 \sim 13 \mathrm{~min}, 40 \% \sim 40 \%$ $585 \mathrm{~B} ; 13 \sim 19 \mathrm{~min}, 40 \% \sim 50.8 \% \mathrm{~B} ; 19 \sim 20 \mathrm{~min}, 50.8 \% \sim 90 \% \mathrm{~B} ; 20 \sim 21 \mathrm{~min}, 90 \% \sim 90 \% \mathrm{~B}$; $58621 \sim 23 \mathrm{~min}, 90 \% \sim 5 \% \mathrm{~B}$. Column temperature maintained at $35^{\circ} \mathrm{C}$; the flow rate was $5870.4 \mathrm{~mL} \cdot \mathrm{min}^{-1}$, with detection wave length of $260 \mathrm{~nm}, 280 \mathrm{~nm}, 320 \mathrm{~nm}$ and injection 588 volume of $2 \mu \mathrm{L}$. Linear regression models between sample concentrationnt and peak 589 area were established to obtain linear regression equation and linear ranges of 590 chlorogenic acid, ferulic acid, senkyunolide I, senkyunolide H, coniferyl ferulate, 591 senkyunolide A, n-butylphthalide, Z-ligustilide, and butylidenephthalide respectively.

\section{Statistical analysis}

593 Statistical analysis was performed in R software (V 2.15.3) (R Foundation for 594 Statistical Computing, Vienna, Austria) and IBM SPSS 20.0 (IBM Corporation, USA). 595 Analysis of variance (ANOVA) and multiple comparison analysis were used to 596 calculate the mean and standard deviation and perform statistical tests. The differences 597 of parameter variance were estimated by ANOVA and Kruskal Wallis rank sum test 598 based on the distribution of parameter statistics. Tukey's honest significant differences 599 tests and Wilcoxon matched-pairs signed-rank test were used for post-hoc comparison. $600 \mathrm{R}$ (V 3.2.2) performed heat map, PCoA, and hierarchical clustering. The R language 601 performed comparison of microorganisms through the 'adonis' function from R's 
602 'vegan' package. The multiple null hypothesis testing of the prior group was performed

603 using the 'anosim' or 'adonis' function from R's 'vegan' package. According to the

604 method of Franzosa et al [52], we performed clustering of differentially abundant

605 features using a custom approach, then the correlation analysis between the

606 differentially abundant chemical components and the differentially abundant

607 microorganisms was carried out (For all clustering analyses, we applied Spearman's

608 rank correlation as a similarity measure with a threshold of $r=0.7)$. Unless otherwise

609 stated, the significance level was set at $p<0.05$.

610 Supplementary Information

611 Additional file 1. Table S1.

612 Additional file 2. Supplementary Figure S1, Table S2. Tentative markers for

613 discriminating A. sinensis samples between every two groups. Note: A, GN and YN

614 group; B, GS and YS group; C, GN and GS group; D, YN and YS group.

615 Additional file 3. Table S3.

616 Acknowledgements

617 We thank Tang YP for useful suggestions, thank Guo ZX for assistance with the

618 seedling samples and granting permission to conduct research in Min Conty Angelica

619 sinensis institute, and we thank our laboratory coworkers from Key Laboratory of

620 Monitoring and Management of Crop Diseases and Pest Insects for their technical

621 support.

622 Authors' contributions 
623 Zhu L and Yan $\mathrm{H}$ participated in the design of the study. Zhu L carried out the

624 experiments, data acquisition, and drafted the manuscript. Yan H, Zhou GS, Jiang CH

625 also contributed to figures generation, literature search, and manuscript editing. Liu P,

$626 \mathrm{Yu}$ G performed the statistical data analysis. Guo S, Wu QN, Duan JA contributed to

627 the discussion of the manuscript. All authors read and approved the final manuscript.

\section{Funding}

629 This work was supported by National Natural Science Foundation of China (No.

630 81773848), Ministry of Finance Central Level of the Special (No. 2060302), 2019

631 Foshan-HKUST Fund (FSUST19-SRI10), and Special Technology System Project for

632 the Modern Agricultural Industry (CARS-21).

\section{Availability of data and materials}

634 The datasets used and/or analysed during the current study are available from the

635 corresponding author on reasonable request. The online version contains datasets

636 available at http://www.ncbi.nlm.nih.gov/bioproject/690235.

637 Ethics approval and consent to participate

638 Not applicable.

639 Consent for publication

640 Not applicable.

641 Competing interests

642 The authors declare that they have no competing interests.

\section{Author details}


$644{ }^{1}$ National and Local Collaborative Engineering Center of Chinese Medicinal Resources 645 Industrialization and Formulae Innovative Medicine, Jiangsu Collaborative Innovation 646 Center of Chinese Medicinal Resources Industrialization, State Administration of 647 Traditional Chinese Medicine Key Laboratory of Chinese Medicinal Resources 648 Recycling Utilization, Nanjing University of Chinese Medicine, Nanjing, China.

$6492^{2}$ Department of Plant Pathology, College of Plant Protection, Nanjing Agricultural 650 University - Key Laboratory of Monitoring and Management of Crop Diseases and Pest 651 Insects, Ministry of Agriculture - Engineering Center of Bioresource Pesticides in 652 Jiangsu Province, Nanjing, China. 


\section{References}

1. Hook ILI. Danggui to Angelica sinensis root: Are potential benefits to European women lost in translation? A review. Journal of Ethnopharmacology. 2014; 152 (1): 1-13.

2. Wei WL, Zeng R, Gu CM, Qu Y, Huang LF. Angelica sinensis in China-A review of botanical profile, ethnopharmacology, phytochemistry and chemical analysis. Journal of Ethnopharmacology. 2016; 190: 116-141.

3. Berendsen RL, Pieterse CMJ, Bakker PAHM. The rhizosphere microbiome and plant health. Trends in Plant Science. 2012; 17 (8): 478-486.

4. Mendes R, Kruijt M, De Bruijn I, Dekkers E, Van Der Voort M, Schneider JHM, et al. Deciphering the rhizosphere microbiome for disease-suppressive bacteria. Science. 2011; 332(6033): 1097-1100.

5. Bulgarelli D, Rott M, Schlaeppi K, Ver Loren van Themaat E, Ahmadinejad N, Assenza F, et al. Revealing structure and assembly cues for Arabidopsis root-inhabiting bacterial microbiota. Nature. 2012; 488(7409): 91-95.

6. Edwards J, Johnson C, Santos-Medellín C, Lurie E, Podishetty NK, Bhatnagar S, et al. Structure, variation, and assembly of the root-associated microbiomes of rice. Proc Natl Acad Sci U S A. 2015; 112(8): E911-920.

7. Walters WA, Jin Z, Youngblut N, Wallace JG, Sutter J, Zhang W, et al. Large-scale replicated field study of maize rhizosphere identifies heritable microbes. Proc Natl Acad Sci U S A. 2018; 115(28): 7368-737.

8. Lakshmanan V, Selvaraj G, Bais HP. Functional soil microbiome: Belowground solutions to an aboveground problem. Plant Physiol. 2014; 166(2): 689-700. 
9. Luo L, Guo C, Wang L, Zhang J, Deng L, Luo K, et al. Negative Plant-Soil Feedback Driven by Re-assemblage of the Rhizosphere Microbiome With the Growth of Panax notoginseng. Front Microbiol. 2019; 10: 1597.

10. Wu L, Chen J, Khan MU, Wang J, Wu H, Xiao Z, et al. Rhizosphere fungal community dynamics associated with Rehmannia glutinosa replant disease in a consecutive monoculture regime. Phytopathology. 2018; 108(12): 1493-1500.

11. Wei X, Wang X, Cao P, Gao Z, Chen AJ, Han J. Microbial Community Changes in the Rhizosphere Soil of Healthy and Rusty Panax ginseng and Discovery of Pivotal Fungal Genera Associated with Rusty Roots. Biomed Res Int. 2020: 8018525.

12. Dong L, Xu J, Zhang L, Cheng R, Wei G, Su H, et al. Rhizospheric microbial communities are driven by Panax ginseng at different growth stages and biocontrol bacteria alleviates replanting mortality. Acta Pharm Sin B. 2018; 8 (2): 272-282.

13. Wu L, Wang J, Huang W, Wu H, Chen J, Yang Y, et al. Plant-microbe rhizosphere interactions mediated by Rehmannia glutinosa root exudates under consecutive monoculture. Sci Rep. 2015; 5: 15871.

14. Li Q, Wu Y, Wang J, Yang B, Chen J, Wu H, et al. Linking Short-Chain N-Acyl Homoserine Lactone-Mediated Quorum Sensing and Replant Disease: A Case Study of Rehmannia glutinosa. Front Plant Sci. 2020; 11: 87.

15. An Z, Guo F, Chen Y, Bai G, Chen Z. Rhizosphere bacterial and fungal communities during the growth of Angelica sinensis seedlings cultivated in an Alpine uncultivated meadow soil. PeerJ. 2020; 8: e8541.

16. Canarini A, Kaiser C, Merchant A, Richter A, Wanek W. Root exudation of primary 
metabolites: Mechanisms and their roles in plant responses to environmental stimuli. Frontiers in Plant Science. 2019; 10: 157.

17. Zhu S, Guo S, Duan JA, Qian D, Yan H, Sha X, et al. UHPLC-TQ-MS coupled with multivariate statistical analysis to characterize nucleosides, nucleobases and amino acids in Angelicae Sinensis Radix obtained by different drying methods. Molecules. 2017; 22(6): 918. 18. De Vos RCH, Moco S, Lommen A, Keurentjes JJB, Bino RJ, Hall RD. Untargeted large-scale plant metabolomics using liquid chromatography coupled to mass spectrometry. Nat Protoc. 2007; 2(4): 778-791.

19. Zhang K, Yan M, Han S, Cong L, Wang L, Zhang L, et al. Identification of Chemical Markers for the Discrimination of Radix Angelica sinensis Grown in Geoherb and Non-Geoherb Regions Using UHPLC-QTOF-MS/MS Based Metabolomics. Molecules. 2019; 24(19): 3536.

20. Zou J, Chen GD, Zhao H, Wang XX, Zhang ZJ, Qu YB, et al. Triangeliphthalides A-D: Bioactive phthalide trimers with new skeletons from: Angelica sinensis and their production mechanism. Chem Commun. 2019; 55(44): 6221-6224.

21. Ma JP, Guo ZB, Jin L, Li YD. Phytochemical progress made in investigations of Angelica sinensis (Oliv.) Diels. Chin J Nat Med. 2015; 13(4): 241-249.

22. Lee HR, Han SI, Rhee KH, Whang KS. Mucilaginibacter herbaticus sp. nov., isolated from the rhizosphere of the medicinal plant Angelica sinensis. Int J Syst Evol Microbiol. 2013; 63(Pt 8): 2787-2793.

23. Han SI, Lee HJ, Lee HR, Kim KK, Whang KS. Mucilaginibacter polysacchareus sp. nov., an exopolysaccharide-producing bacterial species isolated from the rhizoplane of the herb Angelica sinensis. Int J Syst Evol Microbiol. 2012; 62(Pt 3): 632-637. 
24. Kim BC, Poo H, Lee KH, Kim MN, Kwon OY, Shin KS. Mucilaginibacter angelicae sp. nov., isolated from the rhizosphere of Angelica polymorpha maxim. Int J Syst Evol Microbiol. 2011; 62(Pt 1): 55-60.

25. Giacomelli N, Yongping Y, Huber F, Ankli A, Weckerle C. Angelica sinensis (Oliv.) Diels: Influence of Value Chain on Quality Criteria and Marker Compounds Ferulic Acid and ZLigustilide. Medicines. 2017; 4(1): 14.

26. Zhang B, Weston PA, Gu L, Zhang B, Li M, Wang F, et al. Identification of phytotoxic metabolites released from Rehmannia glutinosa suggest their importance in the formation of its replant problem. Plant Soil. 2019; 441(1-2): 439-454.

27. Xin A, Li X, Jin H, Yang X, Zhao R, Liu J, et al. The accumulation of reactive oxygen species in root tips caused by autotoxic allelochemicals - A significant factor for replant problem of Angelica sinensis (Oliv.) Diels. Ind Crops Prod. 2019; 138: 111432.

28. Fierer N, Jackson RB. The diversity and biogeography of soil bacterial communities. Proc Natl Acad Sci U S A. 2006; 103(3): 626-631.

29. Ma F, Wang R, Zhu J, Zhang Y, Wang Y, Hu W, et al. Characterisation comparison of polysaccharides from Dioscorea opposita Thunb. growing in sandy soil, loessial soil and continuous cropping. Int J Biol Macromol. 2019; 126: 776-785.

30. Wu L, Wang J, Wu H, Chen J, Xiao Z, Qin X, et al. Comparative metagenomic analysis of rhizosphere microbial community composition and functional potentials under Rehmannia glutinosa consecutive monoculture. Int J Mol Sci. 2018; 19(8): 2394.

31. Takeuchi M, Sakane T, Yanagi M, Yamasato K, Hamana K, Yokota A. Taxonomic study of bacteria isolated from plants: Proposal of Sphingomonas rosa sp. nov., Sphingomonas pruni sp. 
nov., Sphingomonas asaccharolytica sp. nov., and Sphingomonas mali sp. nov. Int J Syst Bacteriol. 1995; 45(2): 334-341.

32. Expósito RG, Postma J, Raaijmakers JM, De Bruijn I. Diversity and activity of Lysobacter species from disease suppressive soils. Front Microbiol. 2015; 6: 1243.

33. de Bruijn I, Cheng X, de Jager V, Expósito RG, Watrous J, Patel N, et al. Comparative genomics and metabolic profiling of the genus Lysobacter. BMC Genomics. 2015; 16: 991.

34. Kumar M, Revathi K, Khanna S. Biodegradation of cellulosic and lignocellulosic waste by Pseudoxanthomonas sp R-28. Carbohydr Polym. 2015; 134: 761-766.

35. Patel V, Cheturvedula S, Madamwar D. Phenanthrene degradation by Pseudoxanthomonas sp. DMVP2 isolated from hydrocarbon contaminated sediment of Amlakhadi canal, Gujarat, India. J Hazard Mater. 2012; 201-202: 43-51.

36. Silby MW, Winstanley C, Godfrey SAC, Levy SB, Jackson RW. Pseudomonas genomes: Diverse and adaptable. FEMS Microbiol Rev. 2011; 35(4): 652-680..

37. Oteino N, Lally RD, Kiwanuka S, Lloyd A, Ryan D, Germaine KJ, et al. Plant growth promotion induced by phosphate solubilizing endophytic Pseudomonas isolates. Front Microbiol. 2015; 6: 745 .

38. Zhuang L, Li Y, Wang Z, Yu Y, Zhang N, Yang C, et al. Synthetic community with six Pseudomonas strains screened from garlic rhizosphere microbiome promotes plant growth. Microb Biotechnol. 2020.

39. Zhang XH, Lang DY, Zhang EH. Effects of soil sterilization on growth of Angelica sinensis plant and soil microbial populations in a continuous mono-cropping soil. Int J Agric Biol. 2016; 18(2): 458-463. 
40. Yu M, Xie W, Zhang X, Zhang S, Wang Y, Hao Z, et al. Arbuscular mycorrhizal fungi can compensate for the loss of indigenous microbial communities to support the growth of liquorice (Glycyrrhiza uralensis fisch.). Plants. 2020; 9(1): 7.

41. Castrillo G, Teixeira PJPL, Paredes SH, Law TF, De Lorenzo L, Feltcher ME, et al. Root microbiota drive direct integration of phosphate stress and immunity. Nature. $2017 ; 543(7646)$ : $513-518$.

42. Xiong YW, Li XW, Wang TT, Gong Y, Zhang CM, Xing K, et al. Root exudates-driven rhizosphere recruitment of the plant growth-promoting rhizobacterium Bacillus flexus KLBMP 4941 and its growth-promoting effect on the coastal halophyte Limonium sinense under salt stress. Ecotoxicol Environ Saf. 2020; 194: 110374.

43. Bogino PC, Nievas FL, Giordano W. A review: Quorum sensing in Bradyrhizobium. Appl Soil Ecol. 2015;94:49-58.

44. Cai W, Ou F, Staehelin C, Dai W. Bradyrhizobium sp. strain ORS278 promotes rice growth and its quorum sensing system is required for optimal root colonization. Environ Microbiol Rep. 2020; 12(6): 656-666.

45. Nicoletti R, Ferranti P, Caira S, Misso G, Castellano M, Di Lorenzo G, et al. Myrtucommulone production by a strain of Neofusicoccum australe endophytic in myrtle (Myrtus communis). World J Microbiol Biotechnol. 2014; 30(3): 1047-52.

46. Singh S, Pandey SS, Shanker K, Kalra A. Endophytes enhance the production of root alkaloids ajmalicine and serpentine by modulating the terpenoid indole alkaloid pathway in Catharanthus roseus roots. J Appl Microbiol. 2020; 128(4): 1128-1142.

47. Hartmann A, Schmid M, van Tuinen D, Berg G. Plant-driven selection of microbes. Plant and 
Soil. 2009; 321(1-2): 235-257.

48. Sun X, Wang N, Li P, Jiang Z, Liu X, Wang M, et al. Endophytic fungus Falciphora oryzae promotes lateral root growth by producing indole derivatives after sensing plant signals. Plant Cell Environ. 2020; 42(2): 358-373.

49. Huang CM, Chen WC, Lin SH, Wang YN, Shen FT. Exploration of root-associated bacteria from the medicinal plant Platycodon grandiflorum. Microbes Environ. 2019; 34(4): 413-420.

50. Kim YS, Balaraju K, Jeon YH. Biological characteristics of Bacillus amyloliquefaciens AK-0 and suppression of ginseng root rot caused by Cylindrocarpon destructans. J Appl Microbiol. 2017; 122(1): 166-179.

51. Nakaew N, Lumyong S, Sloan WT, Sungthong R. Bioactivities and genome insights of a thermotolerant antibiotics-producing Streptomyces sp. TM32 reveal its potentials for novel drug discovery. Microbiologyopen. 2019; 8(11): 1-7.

52. Franzosa EA, Sirota-Madi A, Avila-Pacheco J, Fornelos N, Haiser HJ, Reinker S, et al. Gut microbiome structure and metabolic activity in inflammatory bowel disease. Nat Microbiol. 2019; 4(2): 293-305.

53. Zhai X, Jia M, Chen L, Zheng CJ, Rahman K, Han T, et al. The regulatory mechanism of fungal elicitor-induced secondary metabolite biosynthesis in medical plants. Critical Reviews in Microbiology. 2017; 43(2): 238-261.

54. You H, Yang S, Zhang L, Hu X, Li O. Promotion of phenolic compounds production in Salvia miltiorrhiza hairy roots by six strains of rhizosphere bacteria. Eng Life Sci. 2018; 18(3): 160-168. 55. Zhai T, Wang Y, Liu C, Liu Z, Zhao M, Chang Y, et al. Trichoderma asperellum ACCC30536 inoculation improves soil nutrition and leaf artemisinin production in Artemisia annua. Acta 
Physiol Plant. 2019; 41(4): 1-11.

56. He C, Wang W, Hou J. Characterization of dark septate endophytic fungi and improve the performance of liquorice under organic residue treatment. Front Microbiol. 2019; 10: 1364.

57. Zhang Y, Chen P, Ye G, Lin H, Ren D, Guo L, et al. Complete Genome Sequence of Pseudomonas Parafulva PRS09-11288, a Biocontrol Strain Produces the Antibiotic Phenazine-1carboxylic Acid. Current Microbiology. 2019; 76(9): 1087-1091.

58. Kakembo D, Lee YH. Analysis of traits for biocontrol performance of Pseudomonas parafulva JBCS1880 against bacterial pustule in soybean plants. Biol Control. 2019; 134: 72-81.

59. Qian Y, Wang Y, Sa R, Yan H, Pan X, Yang Y, et al. Metabolic fingerprinting of Angelica sinensis during growth using UPLC-TOFMS and chemometrics data analysis. Chem Cent J. 2013; 7(1): 42 . 


\section{Figure Legends}

Figure1. Photographs of $A$. sinensis grown in different soil microenvironments. GN (below, A2) and YN (above, A1); GS (below, B2) and YS (above, B1); GN (below, C2) and GS (above, C1) ; YN (below, D2) and YS (above, D1). (Notice: GN represent Gansu non-sterilized group; YN represent Yunnan non-sterilized group; GS represent Gansu sterilized group; YS represent Yunnan sterilized group).

Figure 2. The structure of metabolites and microbial communities of $A$. sinensis samples. PCA scores for the comparison of metabolomic profiles between GN, GS, YN, YS group (A); principal coordinates analysis (PCoA) based on unweighted UniFrac (UUF) distance metric (B); and unweighted pair-group method with arithmetic mean (UPGMA) clustering analyses at phylum level of samples from GN, GS, YN, YS, GNck, GSck, YNck, YSck group (n=6) (C).

Figure 3. Potentially mechanistic associations between rhizosphere microbes and metabolites. Hierarchical clustering heatmap shows a consistent clustering pattern within individual groups and a diverse clustering pattern between different groups (A); covariation between microbes and small molecules in $A$. sinensis, specifically those of differentially abundant microbes and metabolites matched against standards between GS and YS group (Spearman's rank correlation with twotailed nominal $P$ values $)(n=6)(B)$.

Figure 4. The composition of bacteria rhizosphere microbial communities. Shannon index (A); simpson index (B); Phylum distribution (C); heatmap distribution and hierarchical clustering at the genus level (D) of samples from GN, GS, YN, YS, GNck, GSck, YNck, YSck group ( $n=6$ ). 

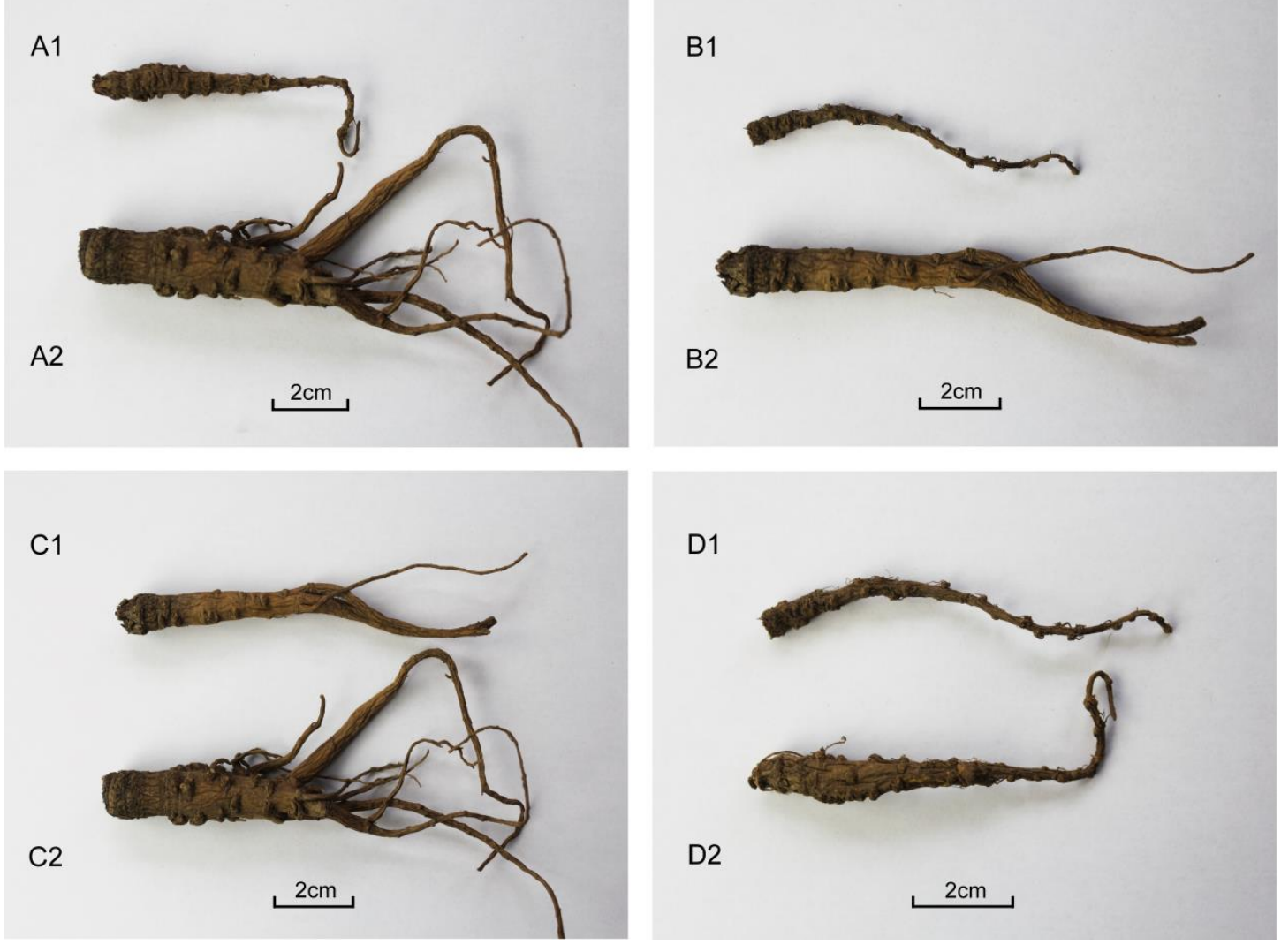

Figure. 1 
A

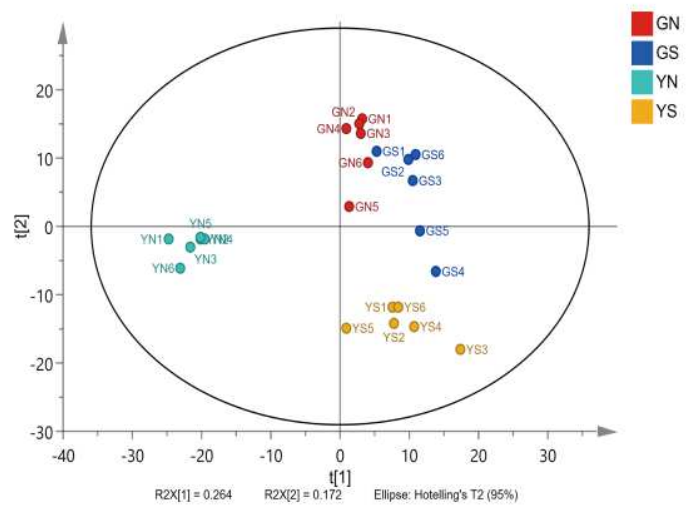

B

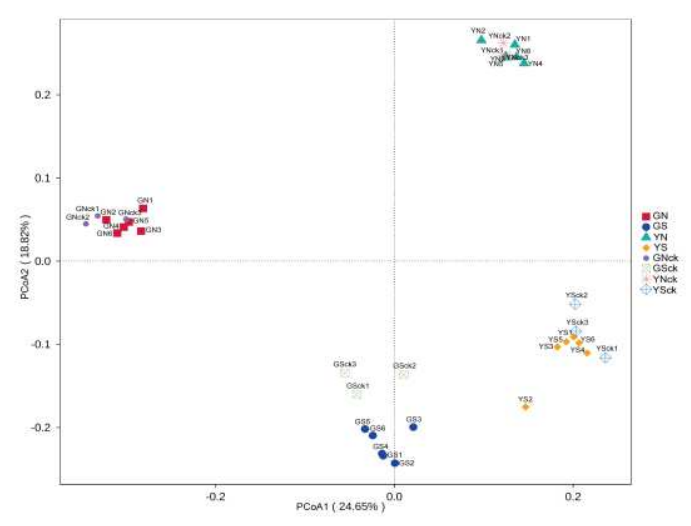

C
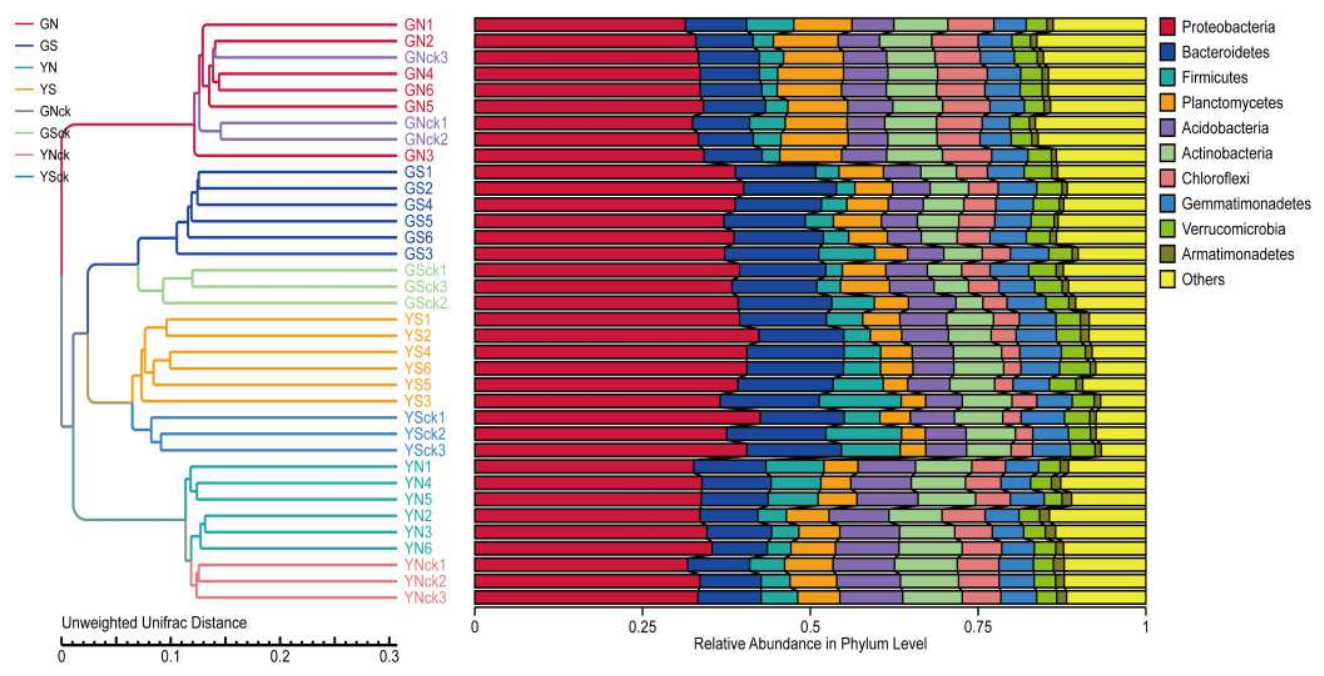

Figure. 2 
A

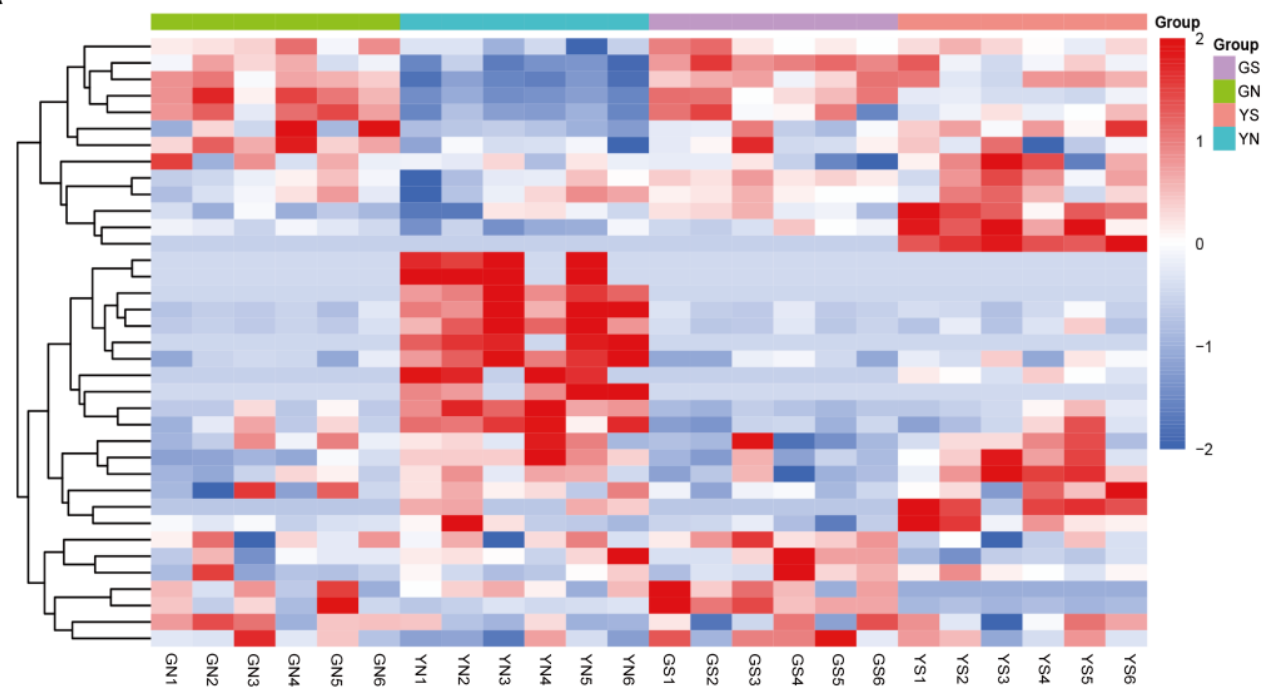

B

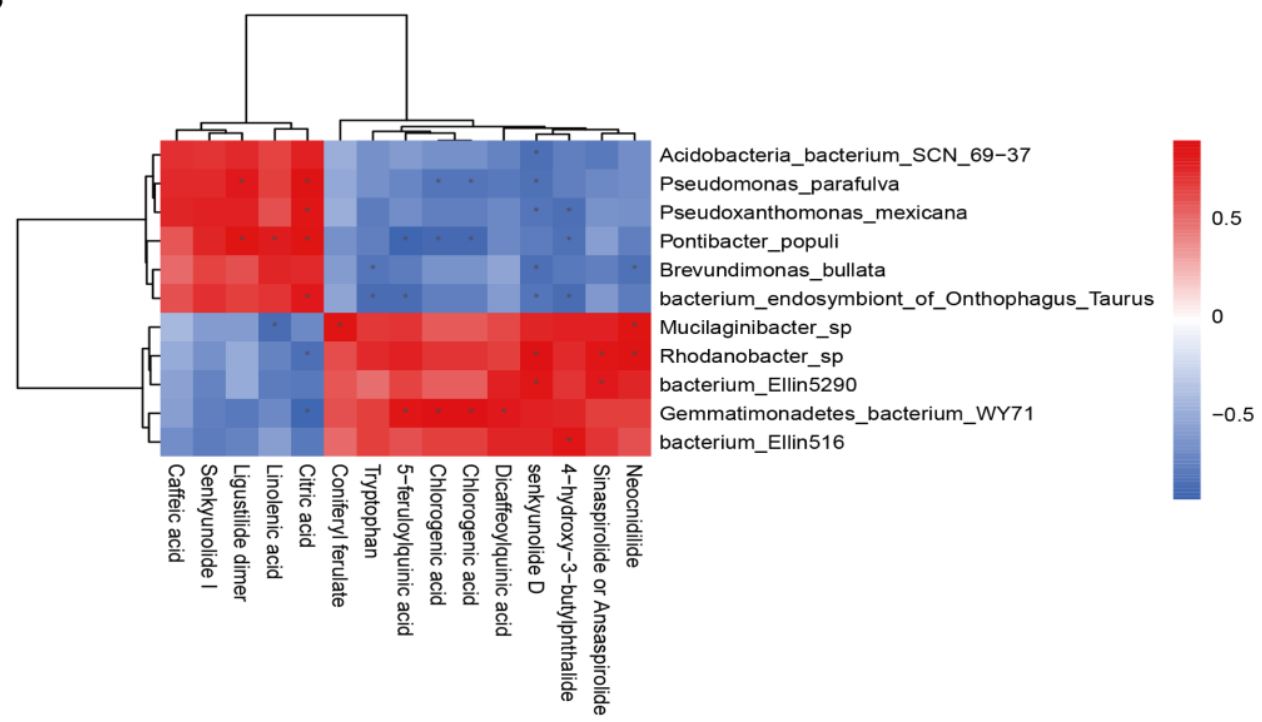

Figure. 3 

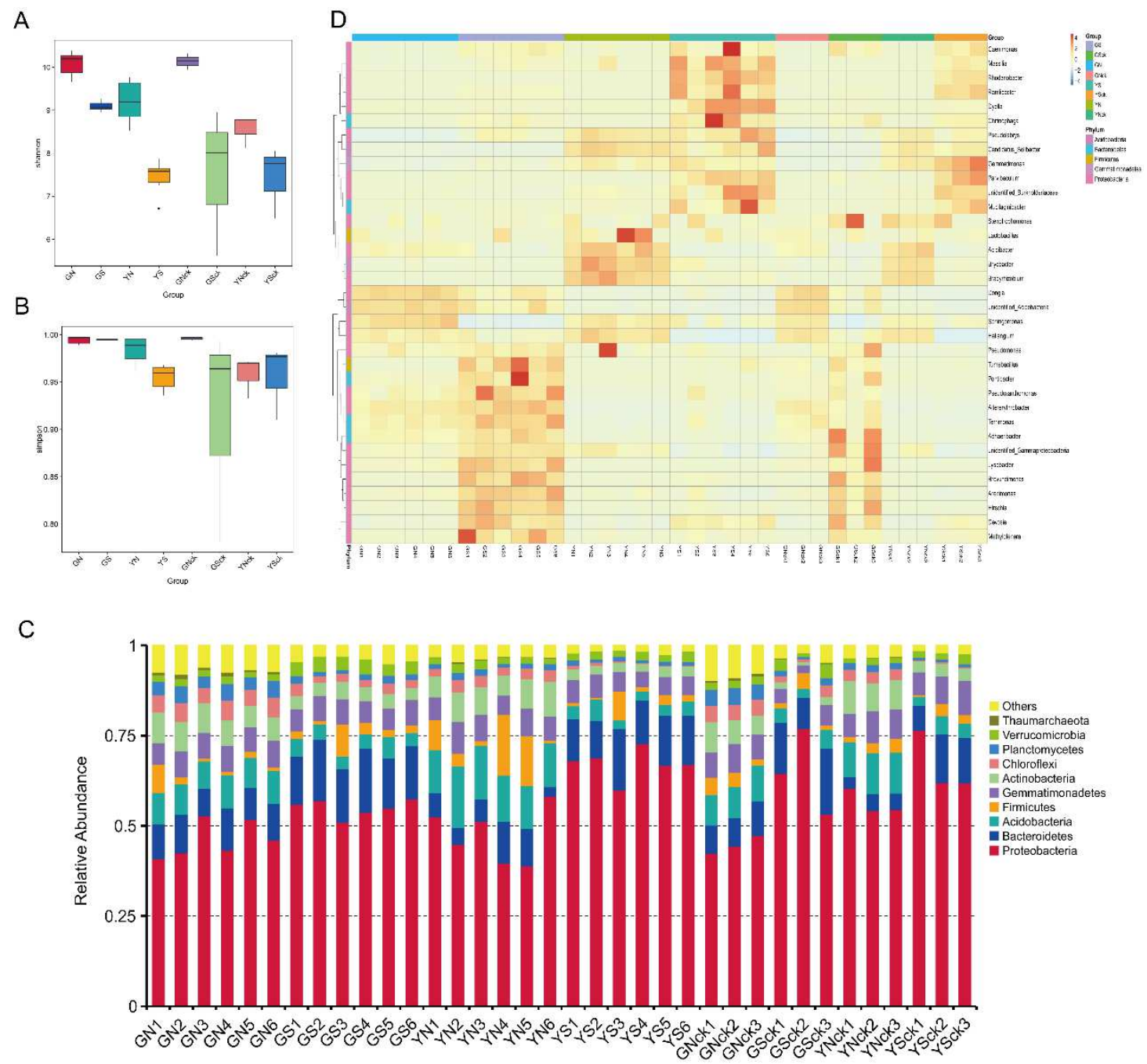

Figure. 4 
Table 1. Root system growth of A. sinensis from different soil microenvironments.

\begin{tabular}{llllll}
\hline Group & $\begin{array}{l}\text { Average } \\
\text { surviving rate } / \%\end{array}$ & $\begin{array}{l}\text { Average dry } \\
\text { weight } / \mathrm{g}\end{array}$ & $\begin{array}{l}\text { Average root } \\
\text { diameter } / \mathrm{mm}\end{array}$ & $\begin{array}{l}\text { Average } \\
\text { rootlet number }\end{array}$ & $\begin{array}{l}\text { Average root } \\
\text { length } / \mathrm{cm}\end{array}$ \\
\hline $\mathrm{GN}$ & $0.77 \pm 0.09 \mathrm{a}$ & $2.94 \pm 0.85 \mathrm{a}$ & $7.86 \pm 1.66 \mathrm{a}$ & $6.94 \pm 1.64 \mathrm{a}$ & $11.14 \pm 2.34 \mathrm{a}$ \\
$\mathrm{YN}$ & $0.63 \pm 0.18 \mathrm{a}$ & $0.88 \pm 0.25 \mathrm{~b}$ & $5.12 \pm 0.88 \mathrm{~b}$ & $1.72 \pm 0.80 \mathrm{~b}$ & $7.65 \pm 0.70 \mathrm{bc}$ \\
$\mathrm{GS}$ & $0.48 \pm 0.15 \mathrm{~b}$ & $1.30 \pm 0.62 \mathrm{~b}$ & $6.14 \pm 1.13 \mathrm{~b}$ & $5.98 \pm 2.01 \mathrm{ab}$ & $10.36 \pm 1.13 \mathrm{c}$ \\
$\mathrm{YS}$ & $0.46 \pm 0.19 \mathrm{~b}$ & $0.36 \pm 0.05 \mathrm{~b}$ & $3.01 \pm 0.74 \mathrm{c}$ & $1.00 \pm 0.00 \mathrm{~b}$ & $9.01 \pm 0.79 \mathrm{~b}$ \\
\hline
\end{tabular}

Values are mean \pm standard error $(n=6)$. Values with the same letter are not statistically signifificantly different, ANOVA, $p<0.05$. 
Table 2. Comparison of the analytes in different groups of $A$. sinensis samples $(\mathrm{mg} / \mathrm{g})$.

\begin{tabular}{|c|c|c|c|c|c|c|c|c|c|}
\hline Group & $\begin{array}{l}\text { chlorogeni } \\
\text { c acid }\end{array}$ & $\begin{array}{l}\text { ferulic } \\
\text { acid }\end{array}$ & $\begin{array}{l}\text { senkyunol } \\
\text { ide I }\end{array}$ & $\begin{array}{l}\text { senkyunol } \\
\text { ide } \mathrm{H}\end{array}$ & $\begin{array}{l}\text { coniferyl } \\
\text { ferulate }\end{array}$ & $\begin{array}{l}\text { senkyunol } \\
\text { ide A }\end{array}$ & $\begin{array}{l}\text { butylphtha } \\
\text { lide }\end{array}$ & $\begin{array}{l}\text { Z- } \\
\text { ligustilide }\end{array}$ & $\begin{array}{l}\text { butylidene } \\
\text { phthalide }\end{array}$ \\
\hline \multirow[t]{2}{*}{ GN } & $1.89 \pm 0.15$ & $0.48 \pm 0.15$ & $0.22 \pm 0.09$ & $0.08 \pm 0.04$ & $0.51 \pm 0.42$ & $0.02 \pm 0.03$ & - & $6.25 \pm 1.34$ & $0.35 \pm 0.08$ \\
\hline & $\mathrm{c}$ & $\mathrm{c}$ & $\mathrm{a}$ & $\mathrm{a}$ & $\mathrm{a}$ & $\mathrm{b}$ & & $\mathrm{b}$ & $\mathrm{a}$ \\
\hline \multirow[t]{2}{*}{ YN } & $3.62 \pm 0.35$ & $0.72 \pm 0.11$ & $0.18 \pm 0.05$ & $0.07 \pm 0.02$ & $0.08 \pm 0.04$ & $0.96 \pm 0.33$ & $0.35 \pm 0.26$ & $9.68 \pm 1.49$ & $0.23 \pm 0.06$ \\
\hline & $\mathrm{a}$ & $\mathrm{b}$ & $\mathrm{a}$ & $\mathrm{a}$ & $\mathrm{b}$ & $\mathrm{a}$ & $\mathrm{a}$ & $\mathrm{a}$ & $\mathrm{b}$ \\
\hline \multirow[t]{2}{*}{ GS } & $2.05 \pm 0.23$ & $0.87 \pm 0.07$ & $0.23 \pm 0.09$ & $0.10 \pm 0.04$ & $0.04 \pm 0.03$ & $0.07 \pm 0.09$ & - & $6.96 \pm 1.52$ & $0.26 \pm 0.09$ \\
\hline & $\mathrm{c}$ & $\mathrm{ab}$ & $\mathrm{a}$ & $\mathrm{a}$ & $\mathrm{b}$ & $\mathrm{b}$ & & $\mathrm{b}$ & $\mathrm{b}$ \\
\hline \multirow[t]{2}{*}{ YS } & $2.88 \pm 0.43$ & $0.97 \pm 0.24$ & $0.04 \pm 0.00$ & $0.01 \pm 0.00$ & $0.10 \pm 0.07$ & $0.32 \pm 0.25$ & $0.03 \pm 0.04$ & $11.01 \pm 2.7$ & $0.07 \pm 0.02$ \\
\hline & $\mathrm{b}$ & $\mathrm{a}$ & $\mathrm{b}$ & $\mathrm{b}$ & b & $\mathrm{b}$ & $\mathrm{b}$ & $8 \mathrm{a}$ & $\mathrm{c}$ \\
\hline
\end{tabular}

Values are mean \pm standard error $(n=6)$. Values with the same letter are not statistically signifificantly different, ANOVA, $p<0.05$.

- Not detected. 


\section{Figures}
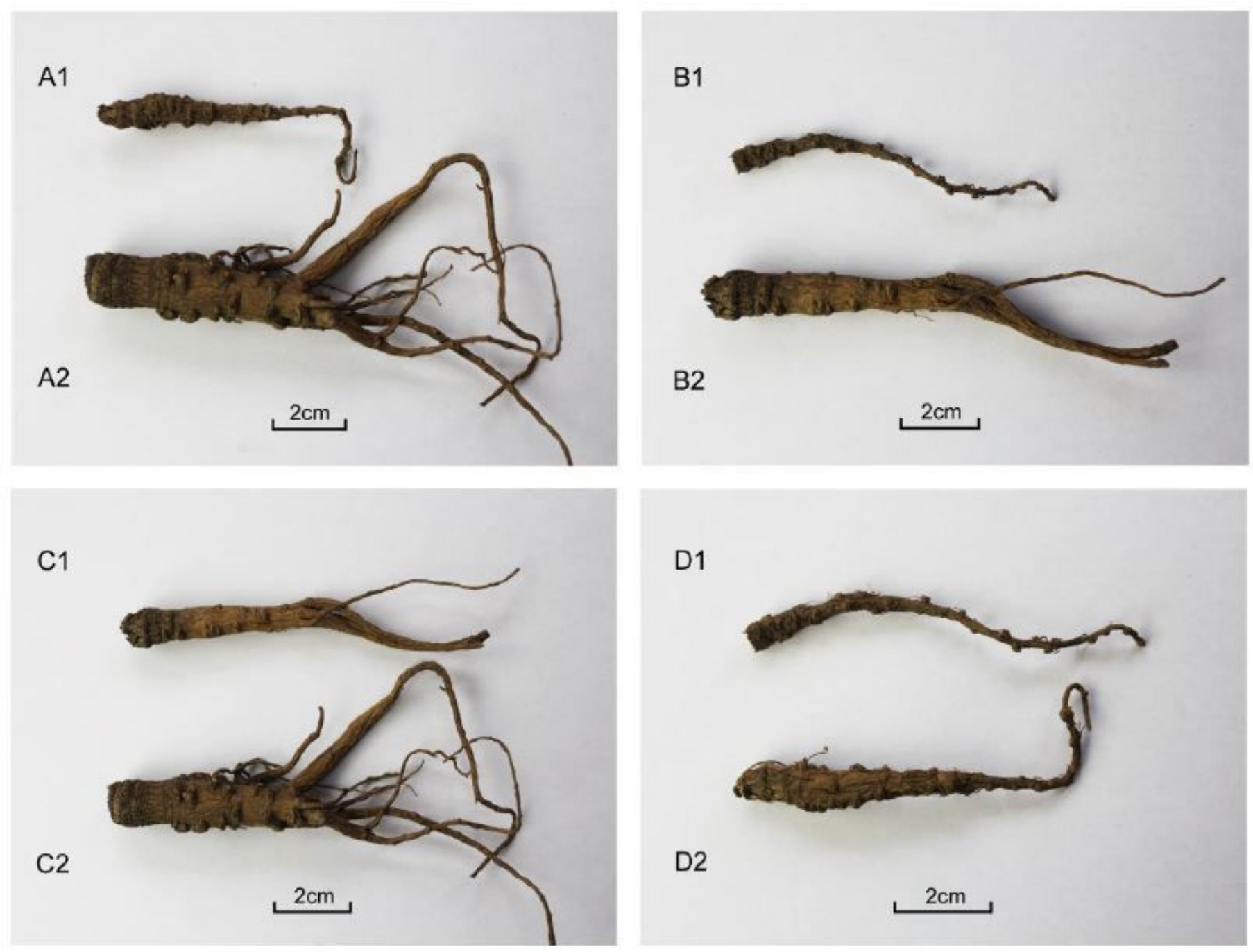

Figure. 1

\section{Figure 1}

Photographs of A. sinensis grown in different soil microenvironments. GN (below, A2) and YN (above, A1); GS (below, B2) and YS (above, B1) ; GN (below, C2) and GS (above, C1) ; YN (below, D2) and YS (above, D1). (Notice: GN represent Gansu non-sterilized group; YN represent Yunnan non-sterilized group; GS represent Gansu sterilized group; YS represent Yunnan sterilized group). 
A

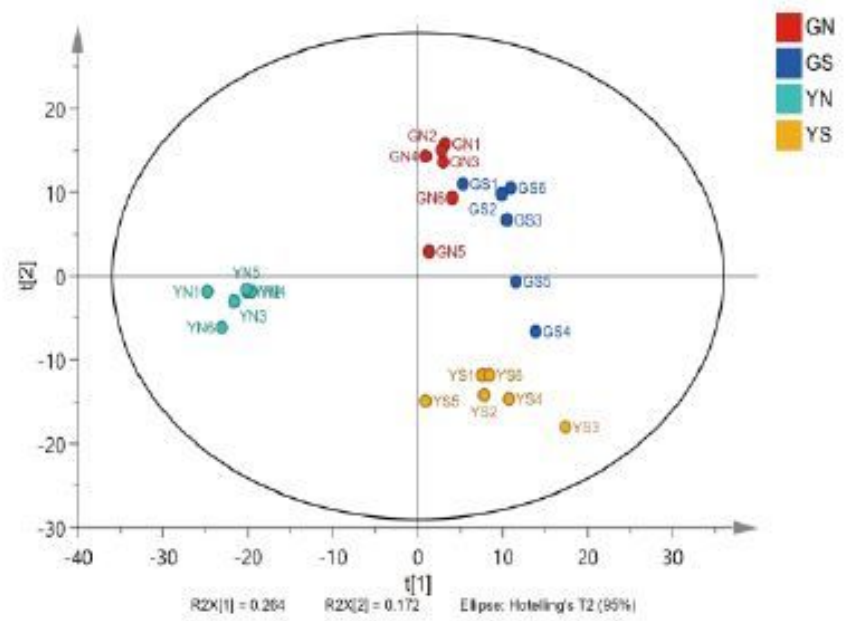

B

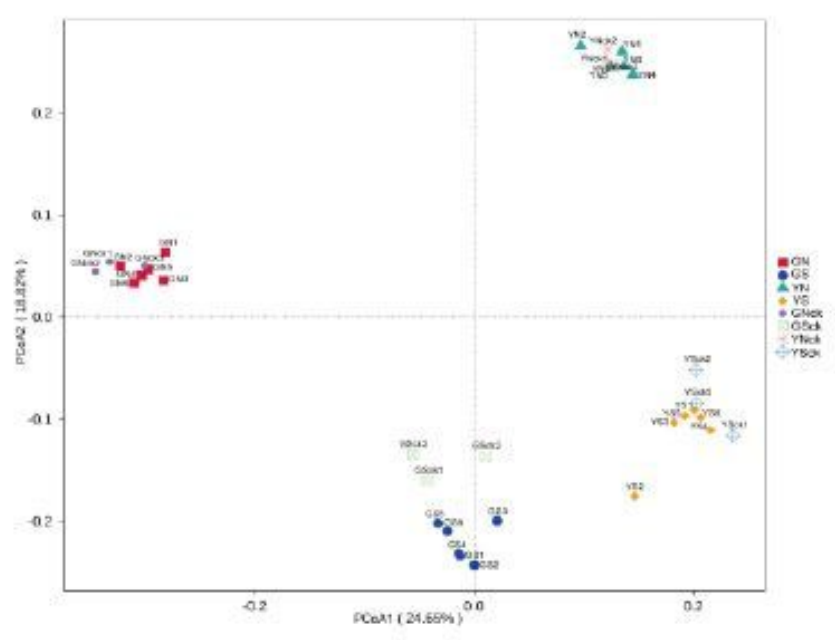

C
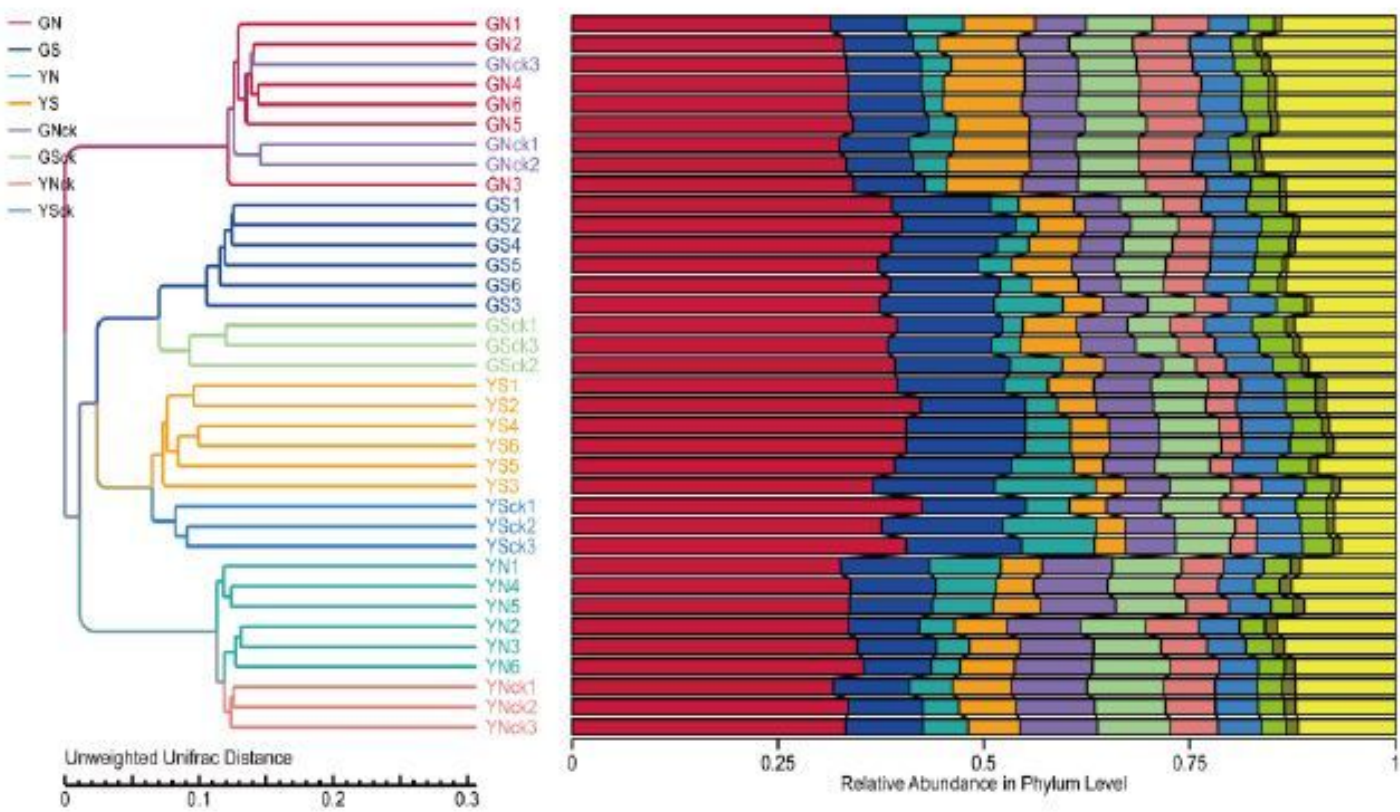

P Proteobacteria Bacteroidetes Firmicules $\square$ Planclomyoetes 口Acidoaacteria $\square$ Actinobacteria $\square$ Chlaraflexi Gemmatimonadetes Verrucomicrotia 1 Armatimonadates $\square$ others

Figure. 2

\section{Figure 2}

The structure of metabolites and microbial communities of A. sinensis samples. PCA scores for the comparison of metabolomic profiles between GN, GS, YN, YS group (A); principal coordinates analysis (PCoA) based on unweighted UniFrac (UUF) distance metric (B); and unweighted pair-group method with arithmetic mean (UPGMA) clustering analyses at phylum level of samples from GN, GS, YN, YS, GNck, GSck, YNck, YSck group $(n=6)(C)$. 
A

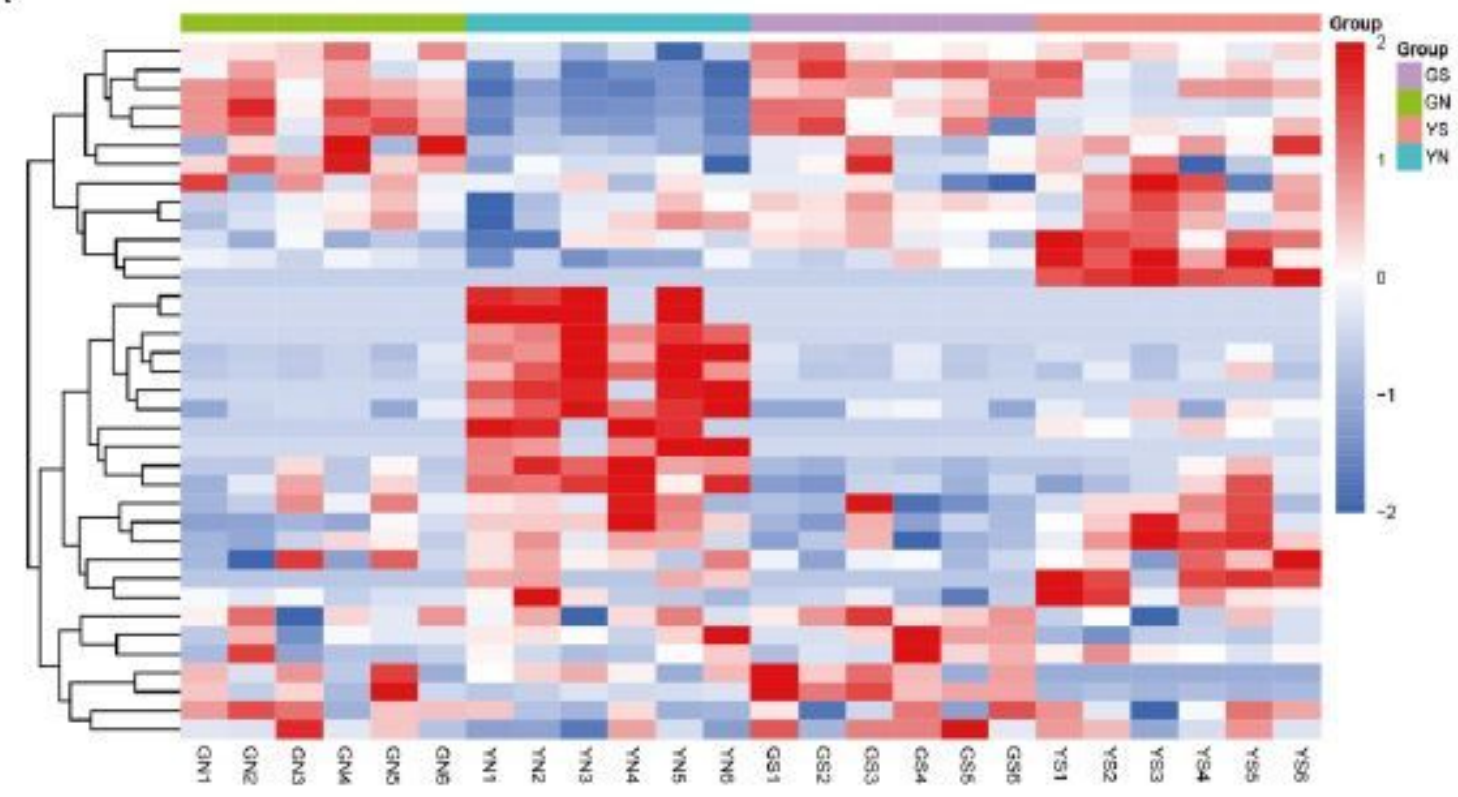

B

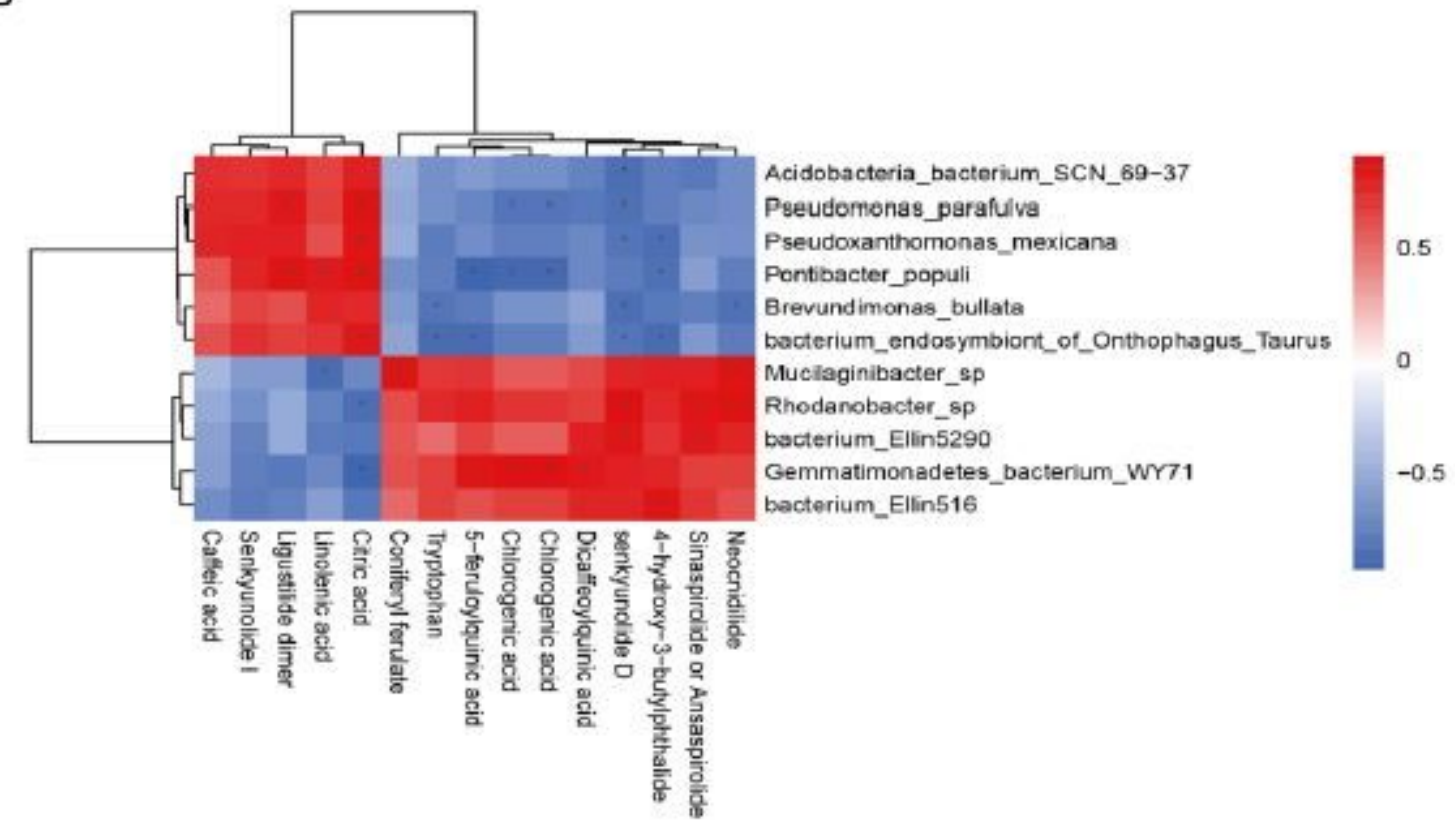

Figure. 3

\section{Figure 3}

Potentially mechanistic associations between rhizosphere microbes and metabolites. Hierarchical clustering heatmap shows a consistent clustering pattern within individual groups and a diverse clustering pattern between different groups (A); covariation between microbes and small molecules in A. sinensis, specifically those of differentially abundant microbes and metabolites matched against standards between GS and YS group (Spearman's rank correlation with two-tailed nominal $P$ values) $(n=6)(B)$. 

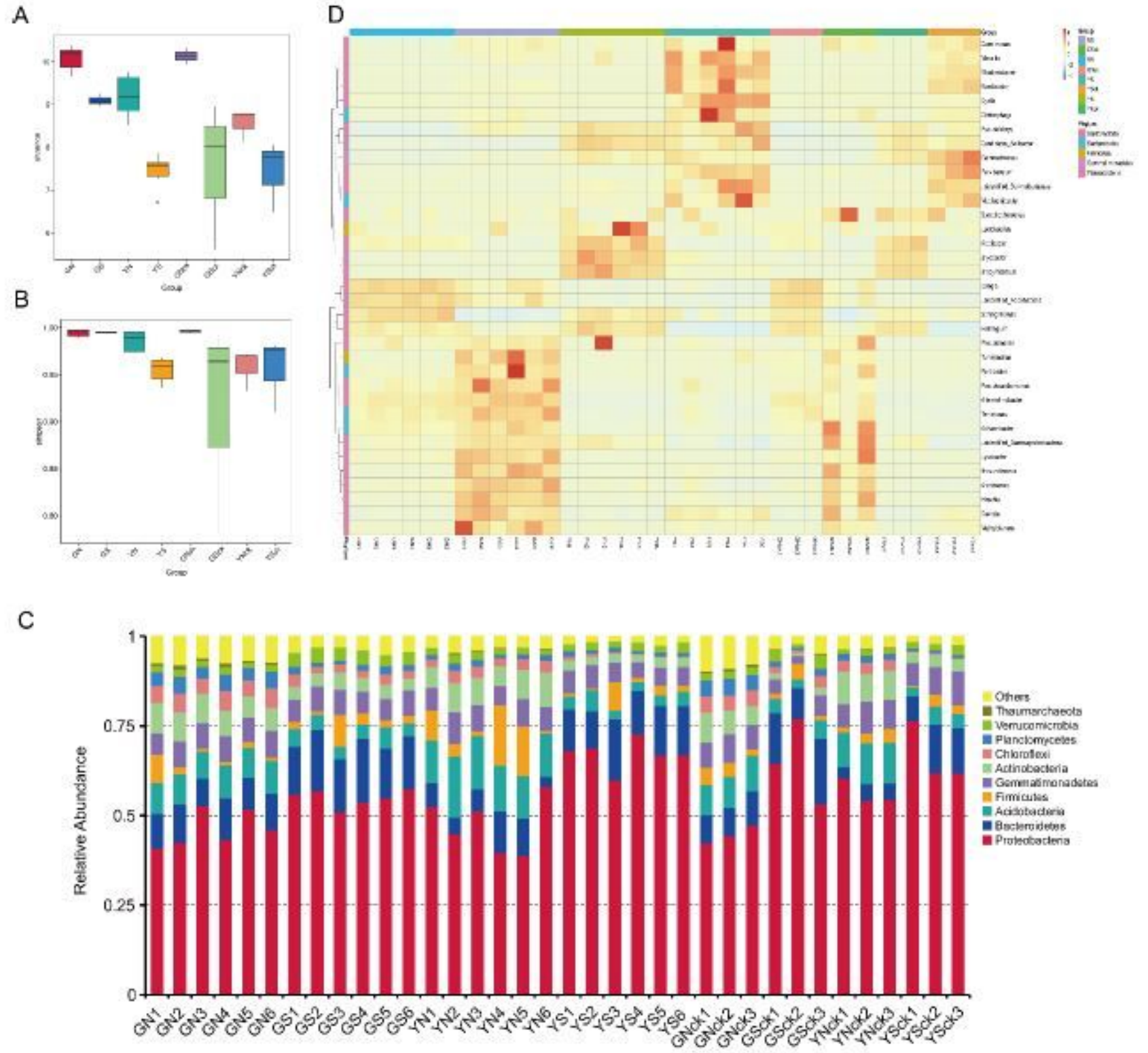

Figure. 4

Figure 4

The composition of bacteria rhizosphere microbial communities. Shannon index (A); simpson index (B); Phylum distribution (C); heatmap distribution and hierarchical clustering at the genus level (D) of samples from GN, GS, YN, YS, GNck, GSck, YNck, YSck group $(n=6)$.

\section{Supplementary Files}

This is a list of supplementary files associated with this preprint. Click to download. 
- FigureS1.tif

- Tables1.docx

- Tables2.docx

- TableS3.docx 